\title{
Current-Mode Network Structures Dedicated for Simulation of Dynamical Systems with Plane Continuum of Equilibrium*
}

\author{
Jiri Petrzela ${ }^{\dagger}$ and Tomas Gotthans \\ Department of Radio Electronics, \\ Brno University of Technology, \\ Technicka 12, 61600 Brno, Czech Republic \\ ${ }^{\dagger}$ petrzelj@feec.vutbr.cz \\ Milan Guzan \\ Department of Theoretical and Industrial Electrical Engineering, \\ Technical University of Kosice, Park Komenskeho 3, \\ 04020 Kosice, Slovak Republic \\ milan.guzan@tuke.sk \\ Received 15 November 2016 \\ Accepted 23 October 2017 \\ Published 29 November 2017
}

\begin{abstract}
This review paper describes different lumped circuitry realizations of the chaotic dynamical systems having equilibrium degeneration into a plane object with topological dimension of the equilibrium structure equals one. This property has limited amount (but still increasing, especially recently) of third-order autonomous deterministic dynamical systems. Mathematical models are generalized into classes to design analog networks as universal as possible, capable of modeling the rich scale of associated dynamics including the so-called chaos. Reference state trajectories for the chaotic attractors are generated via numerical analysis. Since used active devices can be precisely approximated by using third-level frequency dependent model, it is believed that computer simulations are close enough to capture real behavior. These simulations are included to demonstrate the existence of chaotic motion.
\end{abstract}

Keywords: Circuit synthesis; dynamical system; chaos; lumped network; nonlinear dynamics.

\section{Introduction}

Different configurations of lumped analog circuits capable of modeling continuous chaotic dynamics attract significant interest of researchers and engineers for the last

*This paper was recommended by Regional Editor Piero Malcovati.

$\dagger$ Corresponding author.

This is an Open Access article published by World Scientific Publishing Company. It is distributed under the terms of the Creative Commons Attribution 4.0 (CC-BY) License. Further distribution of this work is permitted, provided the original work is properly cited. 
four decades. The reason for this can be found in several unique properties of a strange attractor such as complex geometrical structure, fractal dimension, attractor is dense in a finite state space volume while chaotic waveforms are extremely sensitive to changes of internal system parameters. Since there is no closed-form analytic solution of the describing differential equations, chaotic behavior can be predicted towards neither near nor far future. All these mentioned features cause chaotic oscillators much harder to be practically implemented if compared to conventional analog networks such as amplifiers, modulators, converters, mixers, harmonic or functional generators, etc.

Most existing papers dealing with the construction of chaotic oscillators utilize general design approach based on the concept of analog computers. This method is universal, can be applied to the arbitrary set of the ordinary differential equations and was successfully tested on the mathematical model of jerk function, ${ }^{1-3}$ simplified model of isolated neuron, ${ }^{4}$ general class of autonomous nonlinear systems with smooth and piecewise-linear vector field, ${ }^{5} n$-scroll spiral attractors, ${ }^{6}$ four-dimensional $(4 \mathrm{D})^{7}$ and hyperchaotic systems. ${ }^{8}$ Integrator-based design approach can be easily combined with single or multiple digital feedback two-ports (supplemented by A/D and D/A converters) that implement complex nonlinear transfer function, see Ref. 9 for realization of labyrinth chaos. Another type of chaotic oscillators represents interconnection of higher-order admittance two-terminal element with nonlinear resistor. Pioneering studies in this area have been carried out on famous Chua's oscillator where admittance network is fully passive ${ }^{10-12}$ and individual state variables are voltages across grounded capacitors and current flowing through inductor. Generalization of this design process leads to assumption that structure of the admittance network can realize nonpositive real function if it contains various active elements, check examples given in Refs. 13-15. Chaotic signals can be successfully generated by coupling two harmonic oscillators with the common LC $\operatorname{tank}^{16-18}$ as well as a tank composed of parallel resonant combination of a linear resistor and a frequency dependent negative resistor. ${ }^{19}$ Since fundamental component for the evolution of strange attractor is harmonic, waveform chaotic oscillator can be constructed by slight modification (only single diode is included) of standard oscillator having frequency-dependent passive feedback two-port with losses compensated by amplifier such as Wien-bridge concept. ${ }^{20}$ As a consequence, chaos can be observed as unwanted oscillations in high-Q analog frequency filters like KHN biquadratic filtering section. ${ }^{21}$ Nonlinearity naturally missing in a mathematical description of functional block (filter, amplifier, etc.) can be very simple, ${ }^{22}$ sometimes in the form of amplitude stabilization mechanism. Signum-type nonlinearity responsible for the evolution of chaos can be realized by the logic elements as demonstrated in Ref. 23.

To simplify experimental the verification procedure, voltage-mode circuits are preferred over current-mode flow equivalents. Reason for this is not only in the fact that node voltages can be easily captured by oscilloscope. There is also a much bigger 
platform of integrated active elements that process voltages, which are cheap and widespread over the market stocks. Besides dynamical systems designed by using discrete components, there were successful efforts to construct chaotic oscillators fully- or partially-integrated using available MOS technology. ${ }^{24-27}$ Interesting implementation of grid spiral attractors using Arduino open source integrated development environment is presented in Ref. 28 together with the application of chaos in the selected secure communication issues.

Early ideas about the emergence of chaotic oscillations were closely related to harmonic oscillators where losses in resonant sub-circuit are compensated by using one or several negative resistance elements. ${ }^{29}$ Such structures have single or multiple fixed points with saddle-spiral local vector field geometry and stability index lower than dynamical system dimension. From the viewpoint of chaos generation, it was long time believed that there are always two mechanisms acting in a continuous vector field exhibiting chaos. The so-called stretching property is responsible for the exponential divergence rate of the neighborhood state orbits and is generated by an unstable hyperbolical fixed point; this property creates waveform sensitivity. Second mechanism is trajectory folding and keeps attractor inside a finite volume of the state space. Thus, local instability together with at least one nonlinear scalar function of some state variable should be incorporated into the describing mathematical model. However, this statement has been recently violated by several interesting papers where chaotic systems with completely different formations of equilibrium structure and vector field geometry have been discussed. Please remember that upcoming list of the references is by no way complete survey; it should only help the readers to start their own study on this emerging topic.

The best way to proceed with this study is provided in the fundamental paper, ${ }^{30}$ where many simple chaotic flows are discussed. It is shown that complex dynamical motion is not restricted to the complex mathematical models with many algebraic terms. Reference 31 presents possibility to interchange saddle-spiral equilibrium with saddle-node without a qualitative change of global behavior. Chaotic dynamical system having only one unstable node fixed point is discussed in Ref. 32 and chaotic flow with one nonhyperbolic fixed point is a topic of Ref. 33. Further intensive research demonstrates that chaos is not restricted to dynamical systems with several saddle-type fixed points but also systems having single fixed point with associated real-valued eigenvalues which correspond to three eigenvectors in the local vector filed geometry. Surprisingly, chaotic attractors can also be hidden in the case of the deterministic dynamical systems without equilibrium as shown in Refs. 34-40. Even more interesting discoveries are chaotic systems having only a stable fixed point; for further study, consult Refs. 41-43. One step forward reveals that the scenario for strange attractor evolution can be achieved in dynamical flows with several stable fixed points; see Ref. 44. Surprisingly, deformation of the singular fixed points into higher-dimensional objects does not preclude evolution of a strange attractor. A significant number of research articles have been devoted to the 
mathematical model with chaotic behavior and equilibrium in the form of surface objects such as one or several lines (two parallel),${ }^{45-47}$ hyperbola, ${ }^{48}$ circle $^{49}$ and ellipse, square ${ }^{50}$ other conic-sectioned equilibrium ${ }^{51}$ or a general curve equilibrium. ${ }^{52-54}$ However, it seems that only a portion of line, circle or square is responsible for chaos generation. Based on these recent discoveries in the field of nonlinear dynamic theory, it is not a breathtaking fact that 3D equilibrium structure such as cube can also lead to the evolution of chaos. ${ }^{55}$ Systematic procedure towards the chaotic dynamics with any number of equilibria is described in Ref. 56. Different route-to-chaos scenarios can be observed in the mathematical model of chaotic system with a variable equilibrium. ${ }^{57}$ To end this part of the review procedure for finding arbitrary-dimensional dynamical systems with the chaotic nature can be algorithmized. Suitable form of a starting mathematical model is briefly described in Ref. 58 together with some examples. Chaotic attractors associated with the dynamical system with degenerated equilibria are often referred to as the hidden attractors. ${ }^{59-61}$ It is because the basin of attraction does not include vicinity of equilibrium.

The main motivation for this work is to extend and complete list of the currentmode realizations of chaotic systems published in Ref. 62. Simplicity of models predestinates them for the circuit realizations dedicated for various exhibitions, educational or basic research purposes (for example, bifurcation sequences can be traced and captured).

\section{Mathematical Background}

As already mentioned, the most frequent configuration of vector field with associated chaotic motion is composed of single, two or three fixed points. Typical situation for multi-scroll and multi-grid spiral attractors is symmetrical vector field composed by the repetition of the several affine segments. Trajectory in each segment spirals away from saddle-focus fixed point; there is just one such point per region. Some special cases of mathematical models describing thermo-dynamical systems and chemical reactions do not exhibit fixed points at all. Since the system is closed without external driving forces, it always contains a nonzero energy for time evolution. The main aim of this paper is to address the question if the chaotic dynamics with a nonconventional equilibrium formation can be implemented as hybrid-mode or the fully current-mode electronic circuits (where all state variables are currents). Evidently, strange attractor must be structurally stable and robust to be experimentally observable. The concerned question about robustness of dynamical system remains unanswered in the case of the electronic circuits for signal processing applications (masking, modulation and demodulation) since these do not naturally exhibit equilibrium degenerations.

Consider general mathematical description of a third-order autonomous deterministic dynamical system in the form of first-order ordinary differential equations, 
namely,

$$
\frac{d}{d t} \mathbf{x}=\mathbf{f}(\mathbf{x}), \quad \mathbf{x}=\left(\begin{array}{lll}
x & y & z
\end{array}\right)^{T}, \quad \mathbf{x} \in \Re^{3},
$$

where $\mathbf{x}$ represents a state vector and $\mathbf{f}(\mathbf{x})$ is a continuous smooth nonlinear function. Expected degeneration of the fixed points means that nonlinear problem $\mathbf{f}(\mathbf{x})=\mathbf{0}$ does not lead to the singular solutions but to some plane object; a curve that can be parametrized.

New chaotic dynamical systems can be discovered by using a three-step bruteforce numerical procedure. The first step is the definition of a mathematical model which belongs to general class (1) and has prescribed form of equilibrium and contains only polynomial terms. The second step is the declaration of free parameters of the analyzed mathematical model which will be adjusted during a stochastic optimization routine. The last step is the stochastic optimization sometimes replaced by tabularized calculation of fitness function. However, the dimension of scanned space directly corresponds to the amount of free system parameters and can be significant. Due to the possibility of existence of several attractors (including nonchaotic and trivial solution), the initial conditions should be generated randomly and many times. This approach is capable to uncover attractors excited by equilibrium as well as hidden attractors. Search routine employs repeated computation of some precise and fast-to-be-calculated chaos quantifier such as the largest Lyapunov exponent (LE) obtained from differential equations or small data sets, ${ }^{63-65}$ metric dimension like Kaplan-Yorke or capacity. Nevertheless, the latter case is time consuming and should be applied if parallel processing/computing becomes available. Gradient algorithms are useless because there is no analytic formula for chaos detection. A successive application of this algorithm is demonstrated via few examples in Refs. 66-68. Proposed algorithm can be used for the detection of chaotic motion in the real physical system; both continuous ${ }^{69}$ and discrete.

\subsection{System with line equilibrium}

Speaking in terms of the dynamical motion of lumped electronic circuit, these systems are evolutionary insensitive if the initial conditions accurately satisfy known line equation. This situation leads to the state variables which are frozen with no further time changes of the network quantities. If covering mathematical model is unbalanced by at least one constant term, this line generally need not to cross-over zero, i.e., origin of the state space. If we look on the individual points of this line the associated eigenvalues, the stability indexes and the local geometry can change along this line. Of course, a requirement for dynamical flow to be dissipative is still working.

Two known members form this group of the dynamical systems in which the formula for equilibrium represents a line segment. First can be described by the 
expression

$$
\frac{d x}{d t}=a \cdot z, \quad \frac{d y}{d t}=z \cdot f_{1}(\mathbf{x}), \quad \frac{d z}{d t}=z \cdot f_{2}(\mathbf{x})+\psi(\mathbf{x})
$$

together with a line equation substitution $\psi(\mathbf{x})=b \cdot x-y+c$ leading to a chaotic system with line equilibrium with so far hypothetical form of the scalar functions $f_{1}(\mathbf{x})$ and $f_{2}(\mathbf{x})$. Slightly less general but for upcoming analog circuit design more convenient form is canonical, i.e., without function $f_{2}(\mathbf{x})$

$$
\frac{d x}{d t}=a \cdot z, \quad \frac{d y}{d t}=z \cdot f_{1}(\mathbf{x}), \quad \frac{d z}{d t}=\psi(\mathbf{x}) .
$$

Line equilibrium is the primary subject of interest in contribution. ${ }^{45}$ The presented systems represent alternatives to dynamics given by Eqs. (2) and (3) in the sense there is no linear transformation of coordinates which can form a bridge between these two groups. These dynamical systems can be generalized into class

$$
\frac{d x}{d t}=y, \quad \frac{d y}{d t}=-x+y \cdot f_{1}(\mathbf{x}), \quad \frac{d z}{d t}=-x \cdot f_{2}(\mathbf{x})-y \cdot f_{3}(\mathbf{x}) .
$$

Equilibrium line is given implicitly as one of the coordinate axes of the state space and can be expressed as $\mathbf{x}_{\mathrm{e}}=\left(\begin{array}{lll}0 & 0 z\end{array}\right)^{\mathrm{T}}$. Let us pick up, for example, the first two dynamical systems from a group in Ref. 45. These can be characterized by (4) together with functions

$$
f_{1}(\mathbf{x})=z, \quad f_{2}(\mathbf{x})=1+a \cdot y+b \cdot z, \quad f_{3}(\mathbf{x})=0,
$$

and the numerical values of the system parameters equal $a=15$ and $b=1$. Typical strange attractor can be observed for the initial conditions $\mathbf{x}_{0}=\left(\begin{array}{lll}0.2 & 0 & 0\end{array}\right)^{\mathrm{T}}$ and is demonstrated by means of Fig. 1 . Second dynamical system with line equilibrium can be expressed as

$$
f_{1}(\mathbf{x})=z, \quad f_{2}(\mathbf{x})=a \cdot y+b \cdot z, \quad f_{3}(\mathbf{x})=1
$$

with the internal system parameters equal line $a=17$ and $b=1$. Corresponding strange attractor generated by using the initial conditions $\mathbf{x}_{0}=\left(\begin{array}{lll}0 & 0.4 & 0\end{array}\right)^{\mathrm{T}}$ is given in Fig. 2.

Other member that belongs to this class of the dynamical systems with a single line equilibrium and associated chaotic behavior has auxiliary functions

$$
f_{1}(\mathbf{x})=z, \quad f_{2}(\mathbf{x})=-x+a \cdot y+b \cdot z, \quad f_{3}(\mathbf{x})=1
$$

with the internal system parameters $a=18, b=1$ and the initial conditions $\mathbf{x}_{0}=(0-0.40 .5)^{\mathrm{T}}$. Strange attractor is visualized in Fig. 3. Similarly, line equilibrium is obtained for

$$
f_{1}(\mathbf{x})=z, \quad f_{2}(\mathbf{x})=a \cdot y+b \cdot z, \quad f_{3}(\mathbf{x})=z,
$$




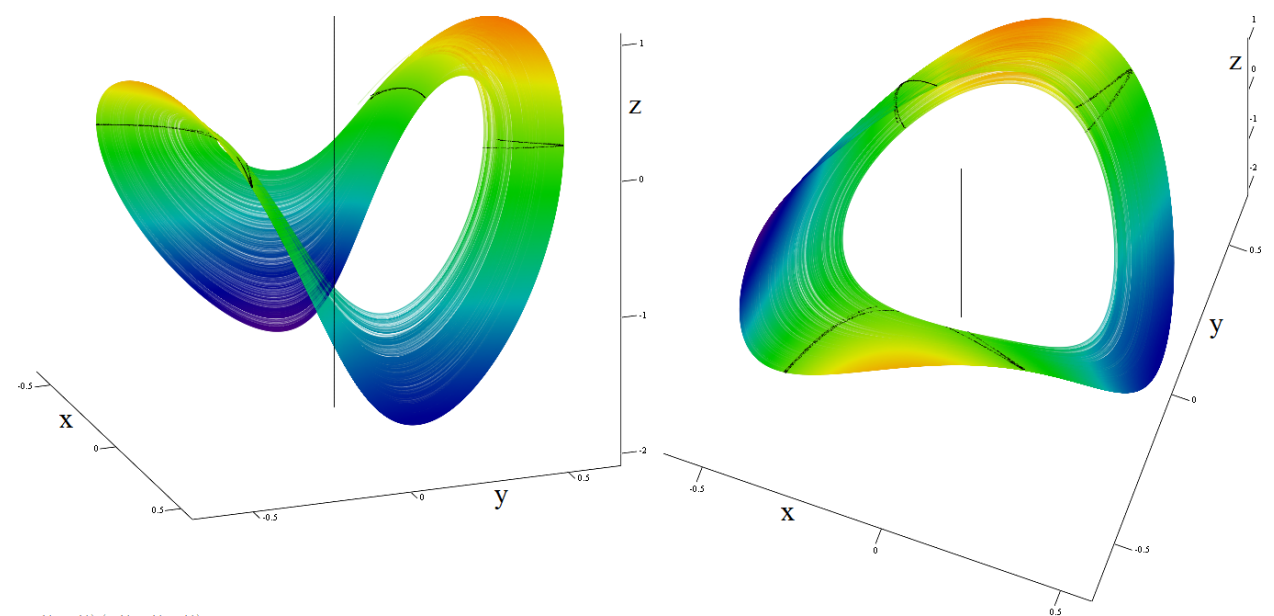

Fig. 1. 3D perspective projections of a typical chaotic attractor observed in the system with line equilibrium given by functions (5a), visualization of equilibrium line and Poincaré section defined by $z=0$.

and chaotic attractor can be observed for the choice of the system parameters $a=4$, $b=0.6$ and a set of the initial conditions $\mathbf{x}_{0}=\left(\begin{array}{lll}0.2 & 0.7 & 0\end{array}\right)^{\mathrm{T}}$ as demonstrated in Fig. 4 .

Finally, the following choice of auxiliary functions

$$
f_{1}(\mathbf{x})=z, \quad f_{2}(\mathbf{x})=y+b \cdot z, \quad f_{3}(\mathbf{x})=-a \cdot y,
$$

also leads to chaos if system parameters are fixed on the numerical values $a=0.04$, $b=0.1$ and a set of the initial conditions is $\mathbf{x}_{0}=\left(\begin{array}{lll}0.8 & 0.8 & 0\end{array}\right)^{\mathrm{T}}$, see Fig. 5 for attractor visualization.

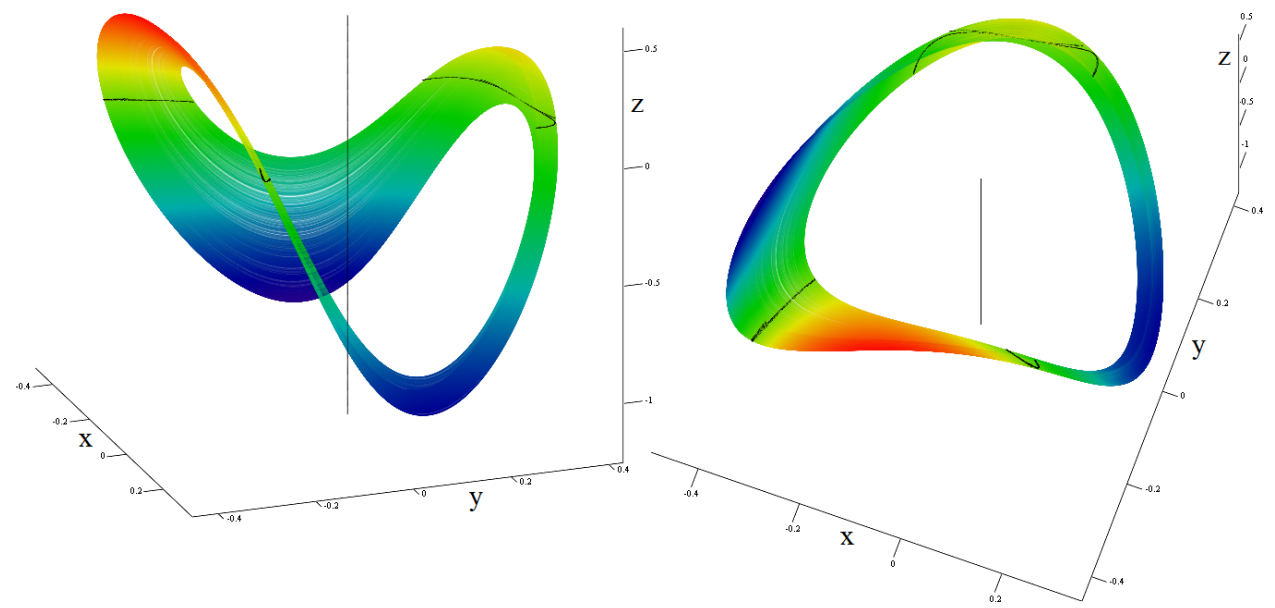

Fig. 2. 3D rainbow-scaled projections of a typical chaotic attractor observed in dynamical system with line equilibrium given by functions (5b), visualization of equilibrium line and Poincaré section given by $z=0$. 


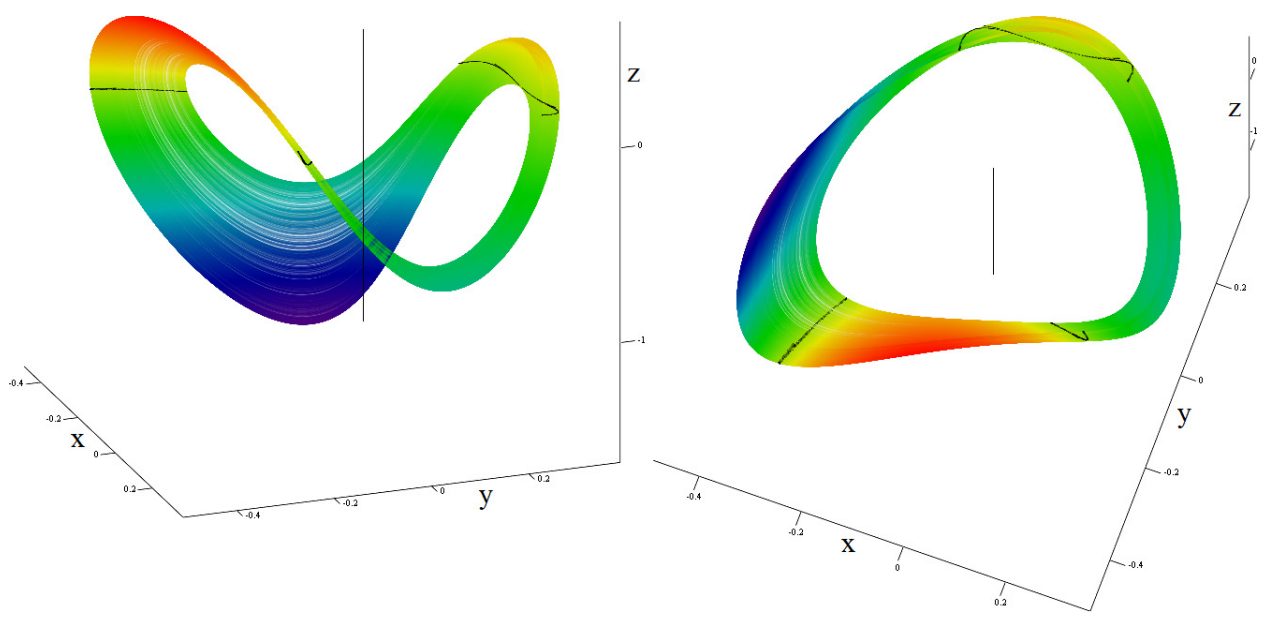

Fig. 3. 3D perspective projections of a typical chaotic attractor observed in system with line equilibrium given by functions (5c), visualization of equilibrium line and Poincaré sections defined by plane $z=0$.

Geometrical structures of the chaotic attractors produced by the dynamical systems with line equilibrium mentioned above are similar. Note that the mathematical model defined by (4) with the additional functions (5a)-(5e) are also closely related. It is because all the cases are discovered using the same numerical approach with the same starting mathematical model with many quadratic terms. ${ }^{70}$ From the viewpoint of practical realization of (4) with terms (5) universal circuit with few switchable linear feedbacks can be constructed. In Ref. 45, there are few dynamical systems that do not directly fit into mathematical model with general description (4)

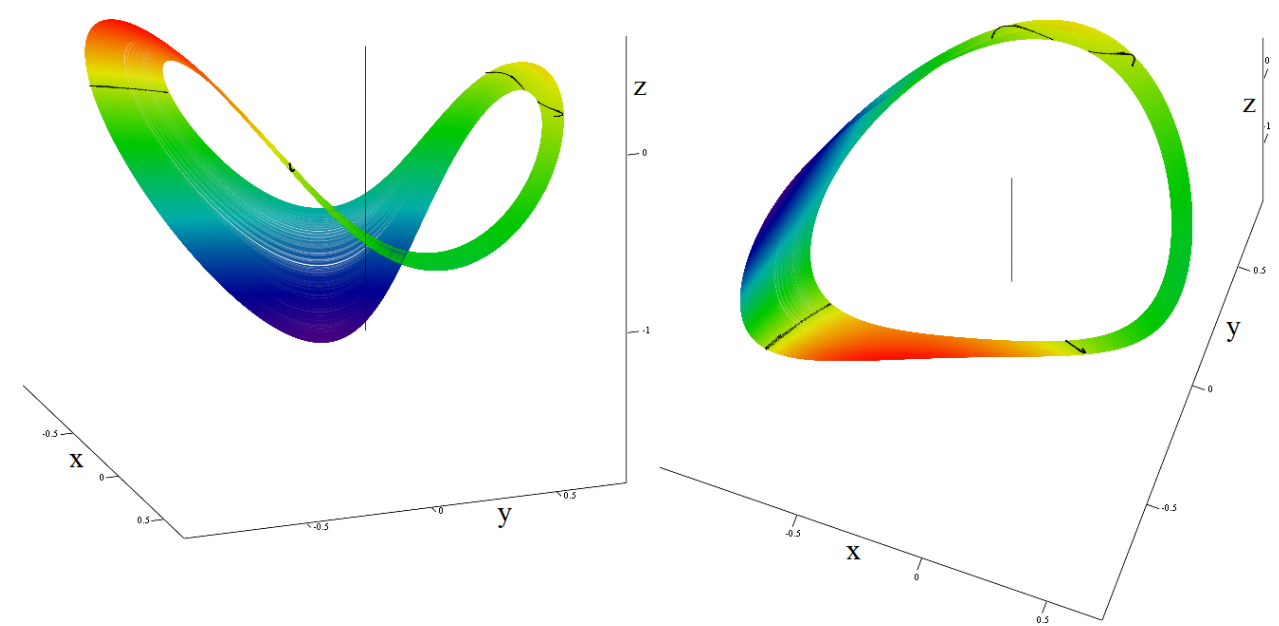

Fig. 4. 3D perspective projections of a typical chaotic attractor observed in dynamical system with line equilibrium given by functions (5d), visualization of equilibrium line and Poincaré sections $z=0$. 


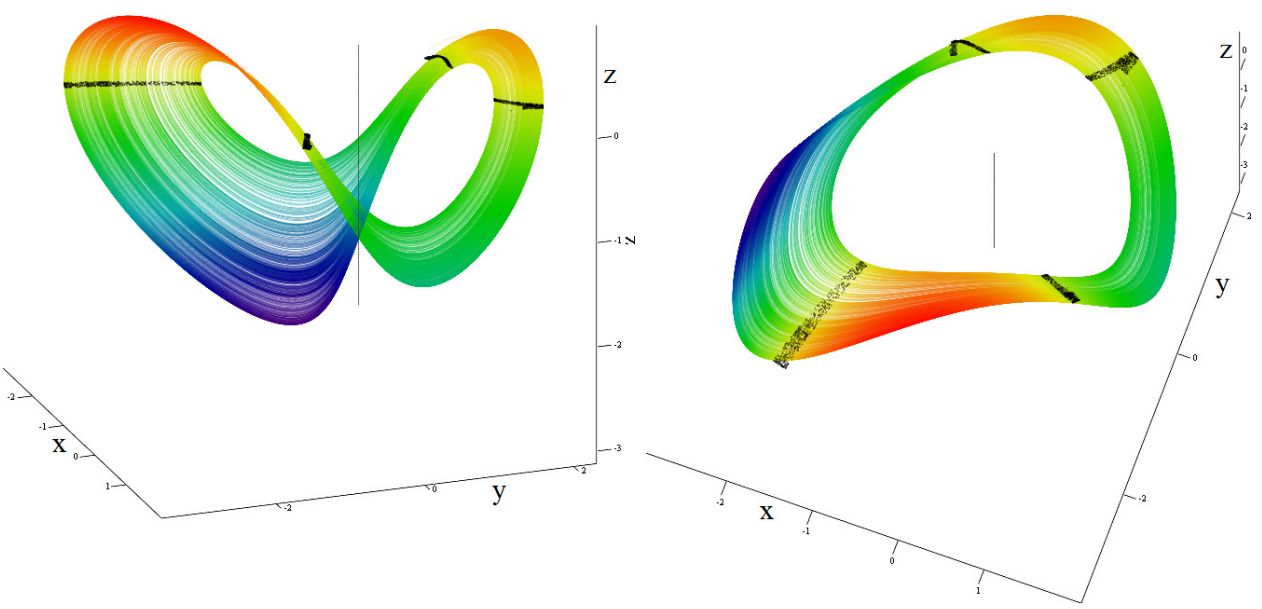

Fig. 5. 3D perspective projections of a typical chaotic attractor observed in the dynamical system with line equilibrium given by functions (5e), visualization of equilibrium line and Poincaré sections $z=0$.

but still preserves line equilibria located on plane $z=0$. However, it can be shown that circuit implementations, either voltage-mode or current-mode, have the same number of the active elements and a final network complexity.

All the dynamical systems defined above are dissipative, i.e., associated dynamics is not time-reversible. Divergence of the flow can be calculated as

$$
\nabla V=f_{1}(\mathbf{x})+y \frac{\partial f_{1}(\mathbf{x})}{\partial y}-x \frac{\partial f_{2}(\mathbf{x})}{\partial z}-y \frac{\partial f_{3}(\mathbf{x})}{\partial z} .
$$

For the system having auxiliary functions (5), we get following volume element exponential contraction rates $\nabla V \approx z-b \cdot x$ for $(5 \mathrm{a})-(5 \mathrm{c})$ and $(5 \mathrm{e})$ and $\nabla V \approx$ $z-b \cdot x-y$ for $(5 \mathrm{~d})$. Examples of the chaotic time-reversible flows are provided in Ref. 71.

Quite recently, chaotic mathematical models with multiple line equilibrium have been proposed. One such example can be found in report ${ }^{47}$ and can be described in general form (2) together with the following functions:

$$
f_{1}(\mathbf{x})=b \cdot y^{2}+c \cdot x \cdot z, \quad f_{2}(\mathbf{x})=-x \cdot y, \quad \psi(\mathbf{x})=y^{2}-1,
$$

and the internal parameters $a=0.6, b=0.3$ and $c=0.5$. Solution $d \mathbf{x} / d t=\mathbf{0}$ considering (2) and (7) leads to a couple of parallel equilibrium lines located at $\mathbf{x}_{\mathbf{e}}=(x \pm 10)^{\mathrm{T}}$, corresponding attractor is shown in Fig. 6 where a set of the initial condition was equal to $\mathbf{x}_{0}=\left(\begin{array}{lll}1 & 0 & 0\end{array}\right)^{\mathrm{T}}$. Note that these lines are infinite in state space while strange attractor is bounded in small volume element such that only a fraction of these lines is responsible for its formation. 


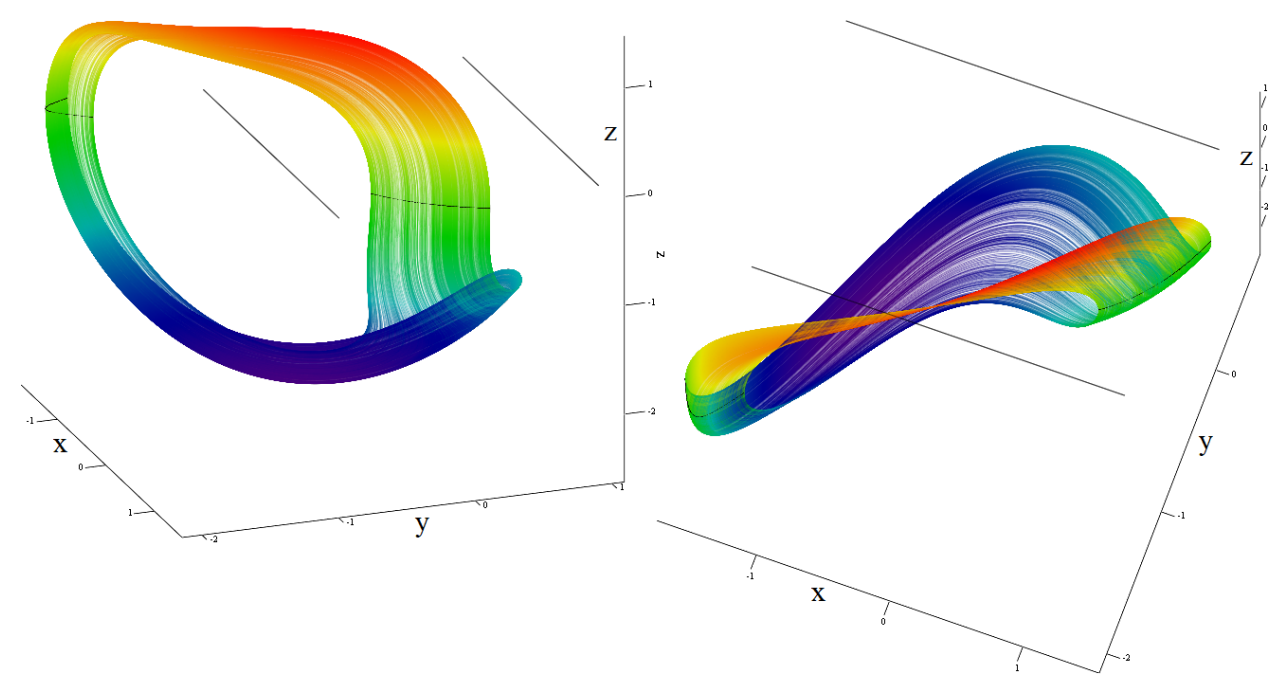

Fig. 6. Chaotic attractor observed in system characterized by functions (7), i.e., with a pair of line equilibrium.

Divergence of flow can be established using formula

$$
\nabla V=z \frac{\partial f_{1}(\mathbf{x})}{\partial y}+f_{2}(\mathbf{x})+z \frac{\partial f_{2}(\mathbf{x})}{\partial z}+\frac{\partial \psi(\mathbf{x})}{\partial z}=2 b \cdot y \cdot z-x \cdot y
$$

Numerical integrations demonstrated in this paper were done by using Mathcad and build-in fourth-order Runge-Kutta method. Final time was chosen to be $t_{\max }=$ 10,000 with a time step $t_{\Delta}=0.01$. Initial conditions can be chosen according to relevant publications where also the bifurcation diagrams, different plots of the Lyapunov exponents versus system parameter and calculated Kaplan-Yorke dimensions can be found. These papers reveal the possibility to see route-to-chaos scenarios via a continuous change of a single constant term. In the circuit practice, this term can be represented by external dc voltage or current control source. By performing this change, the regions of chaos alternate with windows characterized by periodic solution.

\subsection{Model with hyperbolic and parabolic equilibrium}

One logical step further in searching for the chaotic systems with equilibrium located on plane is the hyperbolic and parabolic equilibrium structure. Both the cases are provided in Ref. 47 and a comprehensive study of another different "hyperbolic" case can be found in Ref. 48. The first one can be expressed as (2) with the auxiliary functions

$$
f_{1}(\mathbf{x})=z^{2}-1, \quad f_{2}(\mathbf{x})=y^{2}-z^{2}, \quad \psi(\mathbf{x})=x^{2}-y^{2}-1
$$




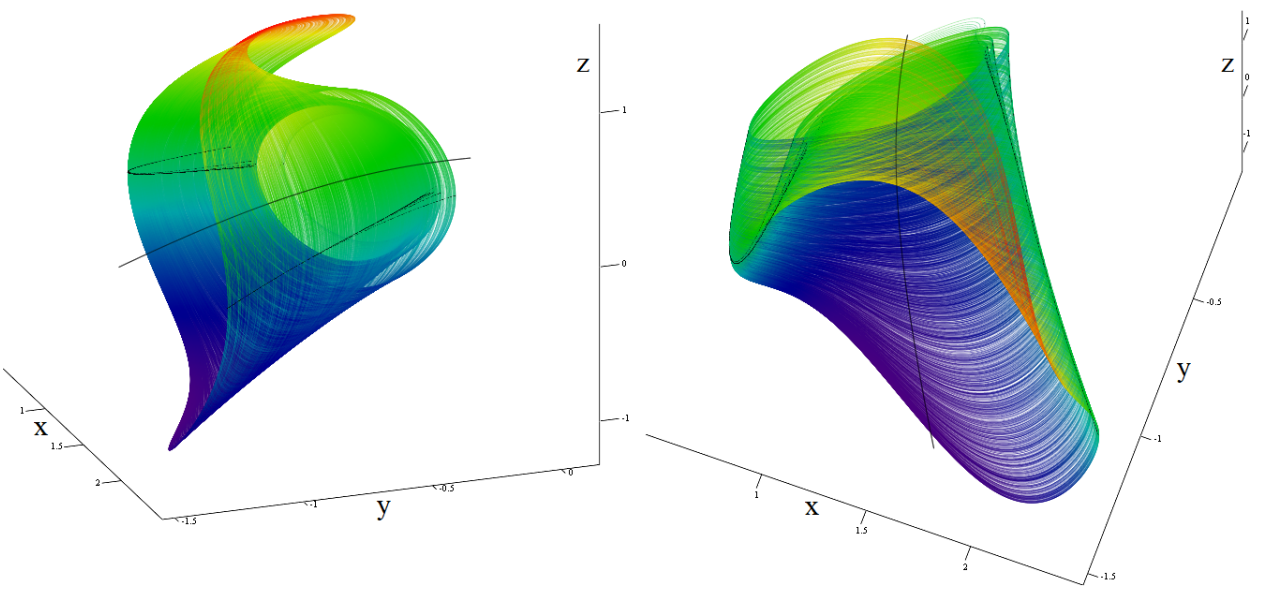

Fig. 7. 3D rainbow-scaled projections of a typical chaotic attractor observed in system having hyperbolic equilibrium, visualization of equilibrium curve and Poincaré section defined by plane $z=0$.

and internal parameter $a=-1$. Corresponding strange attractor arise for a set of the initial conditions $\mathbf{x}_{0}=(0-0.60)^{\mathrm{T}}$ and is illustrated in Fig. 7.

Second dynamical system can be described by general expression (2) with functions

$$
f_{1}(\mathbf{x})=-z^{2}, \quad f_{2}(\mathbf{x})=z-x \cdot y, \quad \psi(\mathbf{x})=x^{2}+y,
$$

and parameter $a=-2$, where interesting strange attractor can be observed if a set of the initial conditions equals $\mathbf{x}_{0}=\left(\begin{array}{lll}0 & 10 & 0\end{array}\right)^{\mathrm{T}}$. Geometrical structure of a corresponding strange attractor is demonstrated using the perspective view in Fig. 8. For both

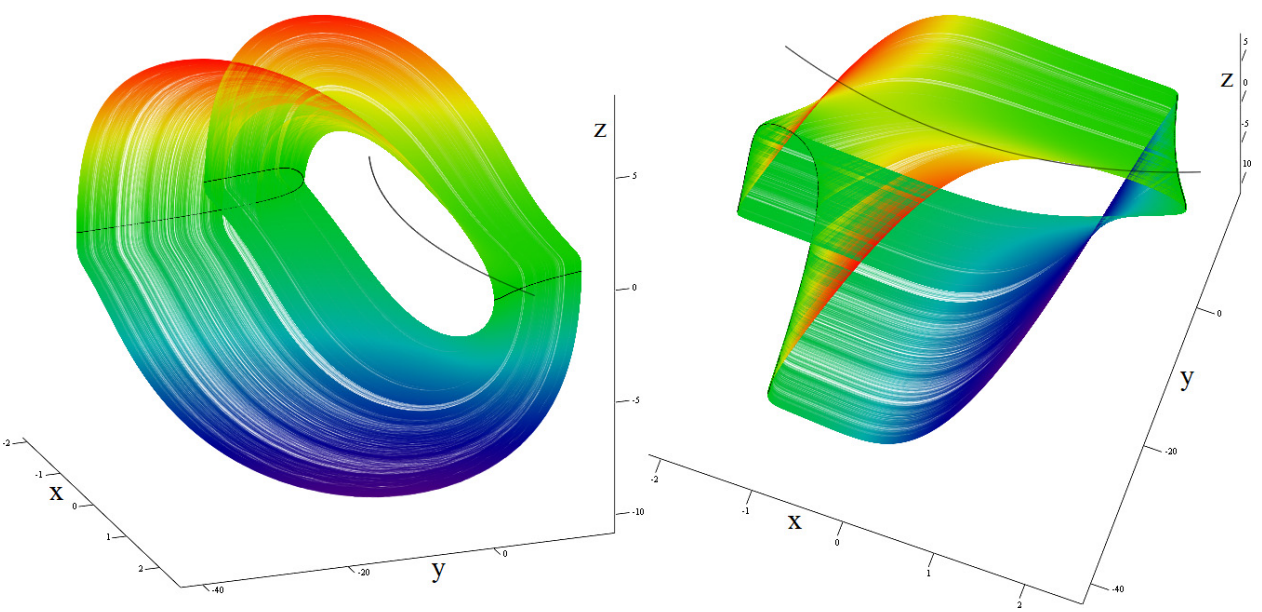

Fig. 8. 3D color-scaled projections of a typical chaotic attractor observed in dynamical system with parabolic equilibrium, visualization of equilibrium curve and Poincaré section given by plane $z=0$. 
systems, equilibrium curve is located on the horizontal plane $z=0$. Note that state attractor of this "parabolic" system occupies large state space volume which can cause problems for circuitry implementation due to the limited dynamical ranges of used active elements.

\subsection{Model having circular and elliptical equilibrium}

Very first example which belongs into class of autonomous dynamical systems having a circular equilibrium has been discovered recently ${ }^{49}$ and can be expressed in form (2). First equation defines a $2 \mathrm{D}$ subspace $(z=0)$ where degenerated equilibrium structure can be found; it is $z=0$ plane again. Equilibrium circle can be unfolded and local bifurcations along this circle can be examined. The third equation covers the implicitly given formula for the equilibrium geometry $\psi(\mathbf{x})$ extended by additional vector field deformation and/or scaling factor $f_{2}(\mathbf{x})$. Desired equilibrium is achieved if $\psi(\mathbf{x})$ is equation of a circle and chaotic behavior can be observed for the remaining functions

$$
f_{1}(\mathbf{x})=b \cdot x-c \cdot z^{2}, \quad f_{2}(\mathbf{x})=-d \cdot x, \quad \psi(\mathbf{x})=x^{2}+y^{2}-r .
$$

Obviously, this mathematical model possesses complementary pair of chaotic attractors. The original one can be observed for a value choice $a=-0.1, b=3$, $c=2.2, d=0.1$ and radius of equilibrium circle $r=1$. Mirrored attractor appears after the trivial inversions of the system coordinates. Note that a vector field ready for the evolution of the chaotic attractor becomes strongly polynomial (up to cubic term) and five four-quadrant analog multipliers will be necessary for the design of this chaotic oscillator. Typical strange attractor generated by this dynamical system in the case of the initial conditions $\mathbf{x}_{0}=\left(\begin{array}{lll}0.3 & 0 & 0\end{array}\right)^{\mathrm{T}}$ is shown in Fig. 9.

In order to define canonical (algebraically simplest) system, a deformation factor can be assumed as zero by reducing the dynamical system (2) into a more practical expression with a single scalar function $f_{1}(\mathbf{x})$ covering polynomial nonlinear terms. Thus, we return to (3). One such example can be described by the following set of auxiliary functions

$$
f_{1}(\mathbf{x})=-b \cdot x \cdot y-y \cdot z+c \cdot z^{2}, \quad \psi(\mathbf{x})=x^{2}+y^{2}-r,
$$

where chaotic motion can be observed for the parameters $a=0.4, b=0.8, c=1.3$ and a set of the initial conditions $\mathbf{x}_{0}=\left(\begin{array}{lll}0.3 & 0 & 0\end{array}\right)^{\mathrm{T}}$, check Fig. 10 for the shape of this strange attractor.

Another example of a dynamical system with circle equilibrium can be found in Ref. 47. This system belongs into class (2) with functions

$$
f_{1}(\mathbf{x})=-y^{2}-x \cdot z, \quad f_{2}(\mathbf{x})=y^{2}-b \cdot z^{2}+x, \quad \psi(\mathbf{x})=x^{2}+y^{2}-r,
$$

and parameters $a=1, b=2$ and radius $r=1$. Corresponding strange attractor for the initial conditions $\mathbf{x}_{0}=\left(\begin{array}{lll}0 & 0.9 & 0.4\end{array}\right)^{\mathrm{T}}$ is provided in Fig. 11. Both attractors are 


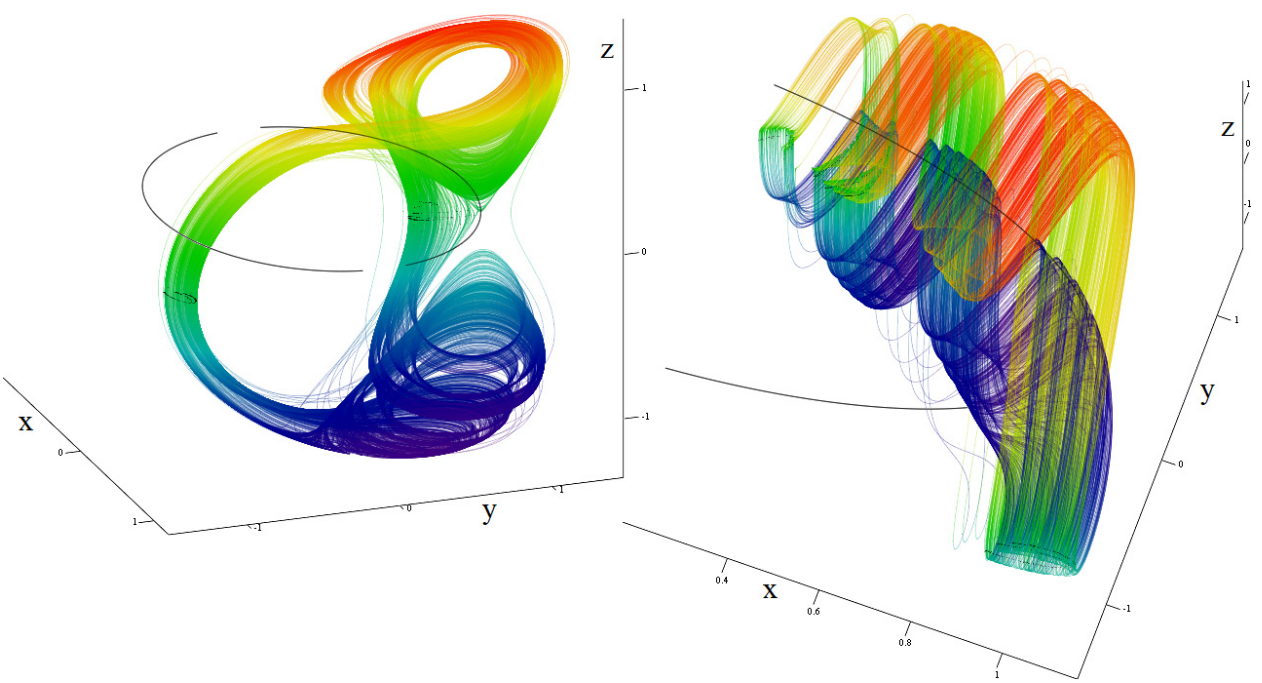

Fig. 9. Perspective rainbow-scaled projections of a typical chaotic attractor observed in dynamical system taken from Ref. 49 with circular equilibrium, visualization of equilibrium line and Poincaré section given by $z=0$.

small in the state space and can be easily realized as the lumped electronic circuits. It should be noted that the above-mentioned systems with circular equilibrium can produce completely dissimilar chaotic attractors with completely different cross-sections.
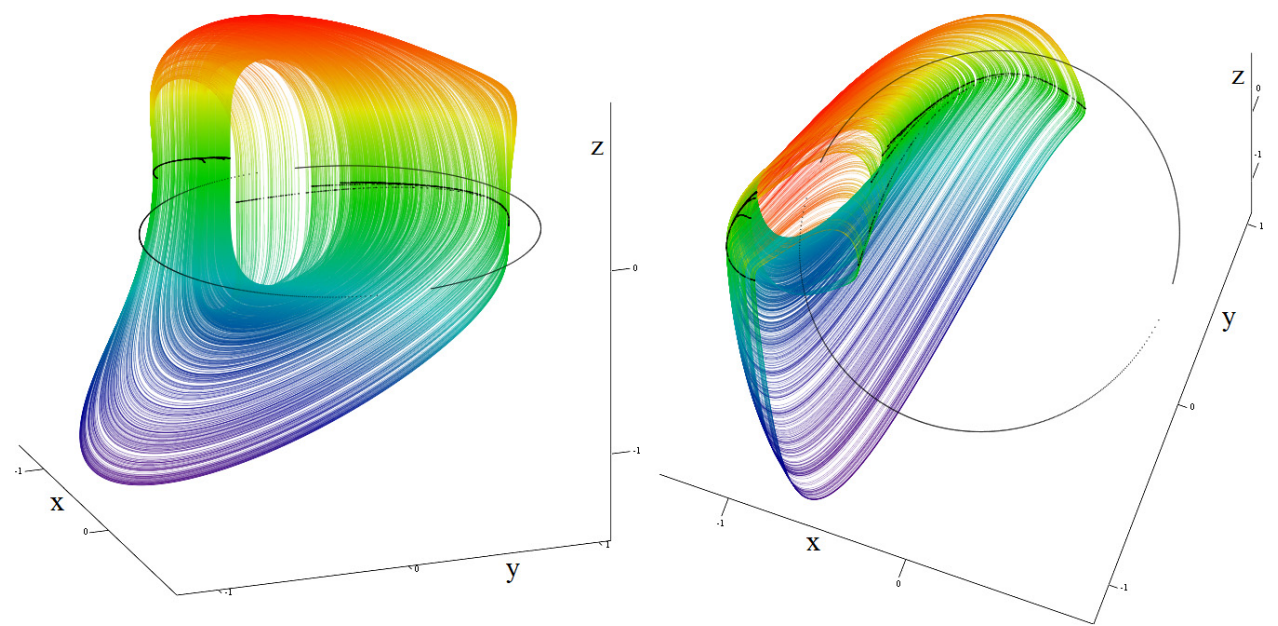

Fig. 10. 3D color-scaled visualization of a typical chaotic attractor observed in system with circular equilibrium taken from Ref. 47, equilibrium structure (black curve) and return map with cross-section $z=0$ (black dots). 


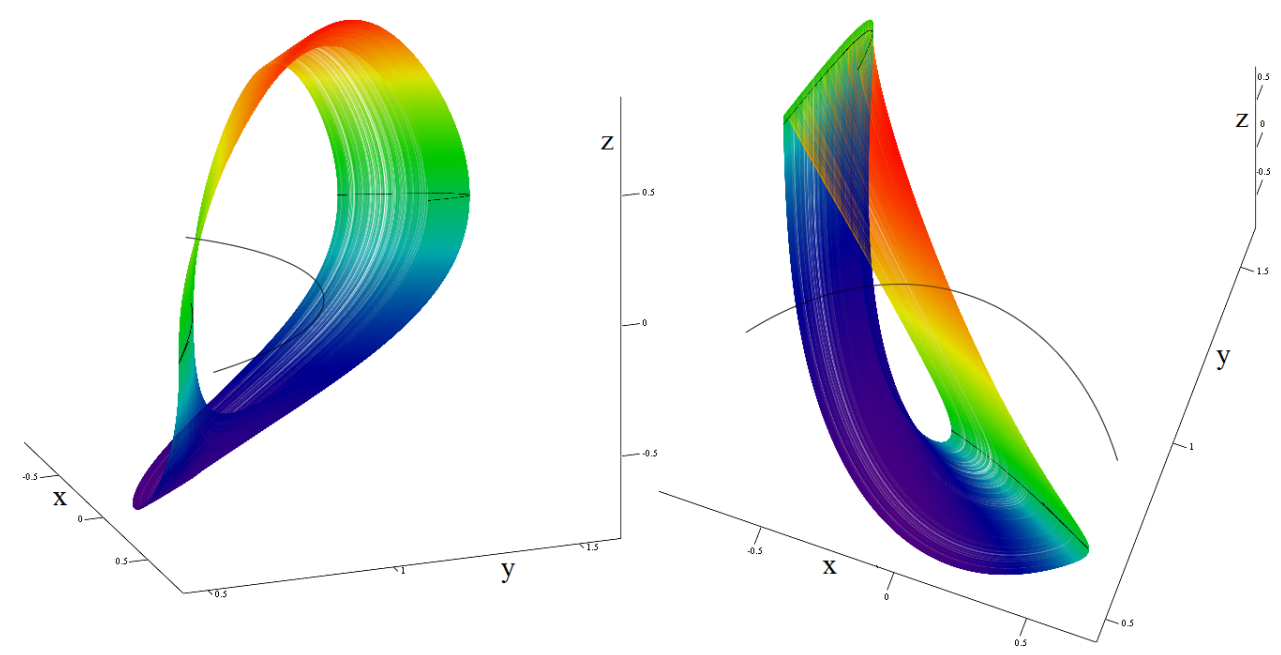

Fig. 11. 3D plot of a typical chaotic attractor observed in a system with circular equilibrium taken from Ref. 47, equilibrium structure (black curve) and return map with cross-section defined by plane $z=0$ (black dots).

Since circle is a special case of ellipse dynamical system described by expression (2) needs only a minor modification to possess elliptical equilibrium, namely,

$$
f_{1}(\mathbf{x})=b \cdot x-c \cdot z^{2}, \quad f_{2}(\mathbf{x})=-d \cdot x, \quad \psi(\mathbf{x})=\left(\frac{x}{r_{1}}\right)^{2}+\left(\frac{y}{r_{2}}\right)^{2}-r,
$$

where variables $r_{1}$ and $r_{2}$ state for minor and major semi-radius of an equilibrium ellipse, respectively. Let us introduce symmetrical ellipse deformation ratio $\sigma=$ $1 / r_{1}=r_{2}$ as arbitrary real positive number. Then, we can make a nonsingular transformation of the coordinates which changes system (2) with functions (10) into same system (2) with functions (13); $x \rightarrow x^{\prime} \cdot \sigma, y \rightarrow y^{\prime} / \sigma, z \rightarrow z^{\prime}$ where $x^{\prime}, y^{\prime}, z^{\prime}$ are the new state variables. Values of the internal parameters associated with (2) and (13) need to be recalculated using simple formulas (results provided for choice $\sigma=5$ ) $a=-0.1 / \sigma=-0.02, b=3 \cdot \sigma^{2}=75, c=2.2 \cdot \sigma=11, d=0.1 \cdot \sigma=0.5$.

For such values, a chaotic attractor is provided by means of Fig. 12 for the initial conditions shifted towards the new basin of attraction, let us pick, for example, $\mathbf{x}_{0}=\left(\begin{array}{lll}0 & 4.5 & 0\end{array}\right)^{\mathrm{T}}$.

Circuit-level simulations again uncover possibility to see the route-to-chaos scenarios via a continuous change of a single model/network parameter, and its value can be adjusted by external dc voltage or current control source. By performing this change, the regions of chaos wander with the windows showing limit cycles having various shapes and periods. The main problem here is that the initial conditions should be precisely adjusted and imposed into the chaotic oscillator, all three state variables at the same time. 


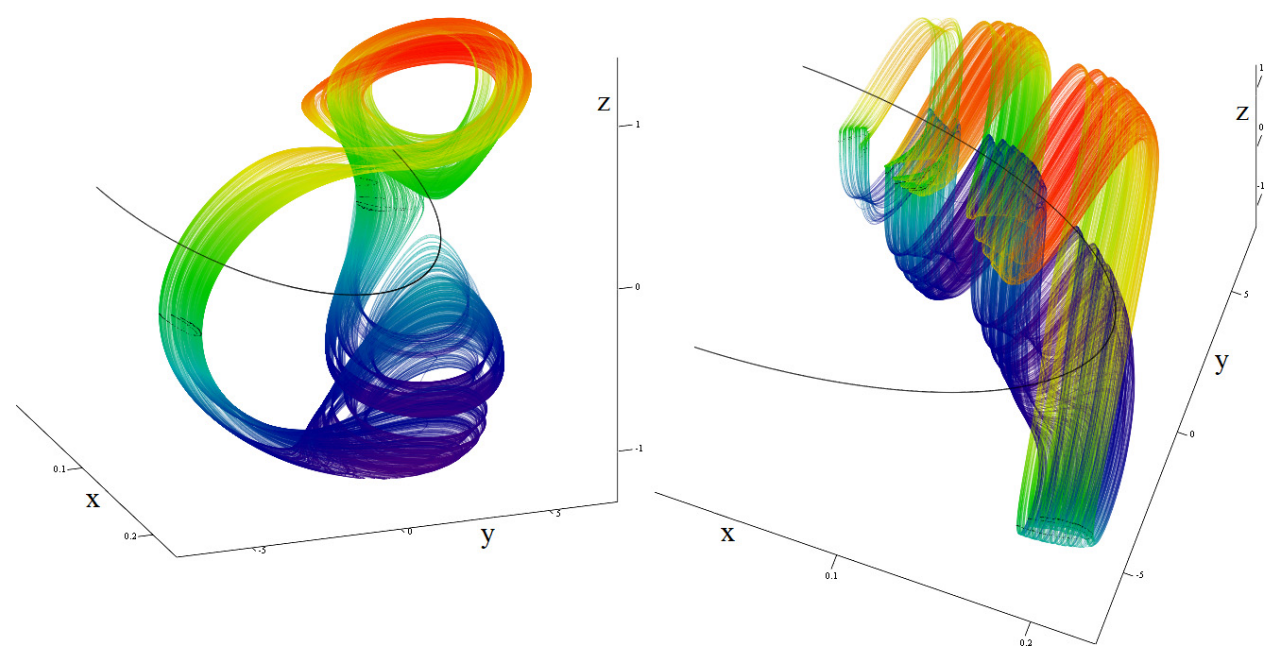

Fig. 12. Perspective rainbow-scaled projections of a typical chaotic attractor observed in a dynamical system with elliptical equilibrium; compare the horizontal axis scales with state space shown in Fig. 9.

\section{Circuitry Implementation and Verification}

The proposed circuit design procedure is based on first Kirchhoff's law applied on nodes with the grounded linear capacitors. This means that each differential equation de facto represents the sum of the currents. Necessary mathematical operations are realized by using active building blocks capable of processing currents instead of voltages. The advantage of such a concept is that the sum of the individual terms in the differential equations can be done by a single node. Typical property of a current-mode realization is low input and high output impedances of used active devices. Some commercially available as well as promising but so far hypothetical active elements dedicated for current-mode signal processing are provided in Ref. 72 .

The most promising active element is a second-generation current conveyor $\left(\mathrm{CCII} \pm\right.$ ) described by three terminal equations $V_{X}=V_{Y}, I_{Y}=0$ and $I_{Z}= \pm I_{X}$. Positive type CCII+ is commercially available under notation AD844 and package also contains output voltage buffer. This device was already implemented for the design of analog chaotic oscillator, see Refs. 73-75. Negative variant CCII- can be found in the markets under notation EL2082; current gain of this device can be adjusted in the range between zero $\left(V_{\text {gain }}=0 \mathrm{~V}\right)$ and two $\left(V_{\text {gain }}=2 \mathrm{~V}\right)$ using external dc voltage source $V_{\text {gain }}$. Multiple-output second generation current conveyors (MOCCII) will be implemented by using appropriate number of CCII+ (each current output requires one active element) to preserve dynamical system ready for immediate design; on the contrary to a brief Ref. 62 where these devices were used without 
hesitation. Well established operational trans-admittance amplifiers with single (OTA) or multiple (MOTA) current outputs are other examples of handy active devices. Currently, both are commercially available (unfortunately limited offer) as the integrated circuits under various denotations such as LM13600, LM13700, CA3080, LT1228, MAX435, MAX436, OPA660 and OPA860 (both with voltage buffer), etc. Last but not least, differential voltage current conveyor (DVCC) can be used if multiple mathematical operations are needed. This device has three inputs with the circuit quantities satisfying $I_{Y 1}=I_{Y 2}=0, V_{X}=V_{Y 1}-V_{Y 2}$ and a single output terminal characterized by $I_{Z}=I_{X}$. More current outputs can be achieved by the connection of several CCII \pm as it is in the case of MOCCII. Thanks to publications, ${ }^{76-78}$ where DVCC has been both designed and applied, this device is no longer hypothetical, although probably not supposed for mass fabrication in the near future. Basic behavior of DVCC can be modeled by input buffers, and standard differential amplifier. Output of this sub-circuit forms node $X$ where some load is supposed to be connected. Current passing through this load can be copied into output $Z$ terminal by using single CCII+. To date, very few research papers have been dedicated to design of the chaotic oscillators with last promising current-mode active element named according to fundamental operation as current differencing transconductance amplifier (CDTA) ${ }^{79-81}$

The reason why modern devices should be considered for design is that it can simplify final network (level of simplification depends on the mathematical model), make one-to-one correspondence between mathematical model parameter and circuit parameter or provide external electronical adjustability of system parameter. The latter advantage will be considered as an essential requirement for the final realization of the chaotic oscillator. In other words, if network topology is chosen correctly, a smooth change of external dc voltages can be used to trace different route-to-chaos scenarios. Moreover, it is still believed that a current-mode signal processing is advantageous from the viewpoint of a better frequency response. Although only theoretically, high frequency currents can be processed without spurious attenuation (without filtering effect of the active elements). In most cases, supply voltage of the current-mode integrated circuits is symmetrical.

The active devices will be first considered close enough to the ideal which means that in the case of current signal processing, input impedance is zero and output impedance is infinite. It means that output current is distributed into the rest of the circuit no matter what kind of load is involved. The next step is that input impedance is modeled by basic resistance connected in series with inductance while output impedance is composed by resistance with high-frequency value degradation caused by capacitor connected in parallel.

It should be noted that many upcoming network realizations do not contain resistors and are suitable for full on-chip implementations using the common CMOS technology. 


\subsection{Chaotic oscillators}

Circuit synthesis belongs to tasks having multiple correct solutions. Design engineer can construct many completely different analog circuits that behave identically as a given mathematical model. Some of these realizations can be celebrated because of simplicity, the others can benefit from the minimal number of elements and the rest of realizations can have the one-to-one relations between model parameters and circuit variables. Remember that we are focused on the current-mode circuits only; it means that all state variables are currents. In some realizations of chaotic oscillators, initial conditions need to be imposed into proper network branches. This requirement can be rather problematic to satisfy in the case of current-mode operational regime. Here, voltage-mode is probably the better idea.

\subsubsection{Oscillator with line equilibrium}

Dynamical system with general line equilibrium expressed as (2) can be modeled by a current-mode network provided in Fig. 13. Covering differential equations are as follows:

$$
\begin{aligned}
& \frac{d i_{x}}{d t}=\frac{g_{m 1}}{C_{1}} \cdot i_{z}, \quad \frac{d i_{y}}{d t}=\frac{g_{m 2}}{C_{2}} \cdot \varepsilon_{1} \cdot i_{z} \cdot f_{1}\left(i_{x}, i_{y}, i_{z}\right), \\
& \frac{d i_{z}}{d t}=\frac{g_{m 3}}{C_{3}}\left[\varepsilon_{2} \cdot i_{z} \cdot f_{2}\left(i_{x}, i_{y}, i_{z}\right)+\gamma_{1} \cdot i_{x}-i_{y}+\frac{V_{c}}{R_{c}}\right],
\end{aligned}
$$

where $f_{1}\left(i_{x}, i_{y}, i_{z}\right)$ and $f_{2}\left(i_{x}, i_{y}, i_{z}\right)$ are fully current-mode nonlinear scalar transfer functions, $\varepsilon_{k}$ is transfer factor of $k$ th current multiplier and $\gamma_{i}$ is multiplication factor of $i$ th CCII-. Quite common situation is that the required current gain factor $\gamma_{i}$ of some multiplier is much bigger than that allowed by a manufacturer of EL2082. This obstacle can be removed simply by a cascading sufficient number of CCII-. This interconnection is done by using $Z \rightarrow X$ current terminals. Simultaneously, it is assumed that reaching output- $Z$ current saturation is out of question for the employed conveyors finishing cascade similarly as going out of dynamical range for linear operation which is allowed for input- $X$ current. Since integrated analog multiplier EL4083 has balanced current outputs and only one is actually used circuit designer should take care of remaining output; i.e., connect optimal-valued resistor to terminate unused output.

For each designed oscillator, working capacitors can be chosen the same $C_{1}=$ $C_{2}=C_{3}$ as well as resistors $R_{1}=R_{2}=R_{3}$ without the loss of generality; for the frequency components falling into acoustic range time constant $\tau=R \cdot C=$ $10^{4} 10^{-7}=1 \mathrm{~ms}$ has been chosen. External DC voltage $V_{c}$ represents equilibrium line offset and can be both positive and negative. However, a slight change of an equilibrium structure can dramatically influence observed state attractor. Of course, $V_{c}$ and $R_{c}$ together with associated CCII+ can be interchanged by DC source of a constant current. 


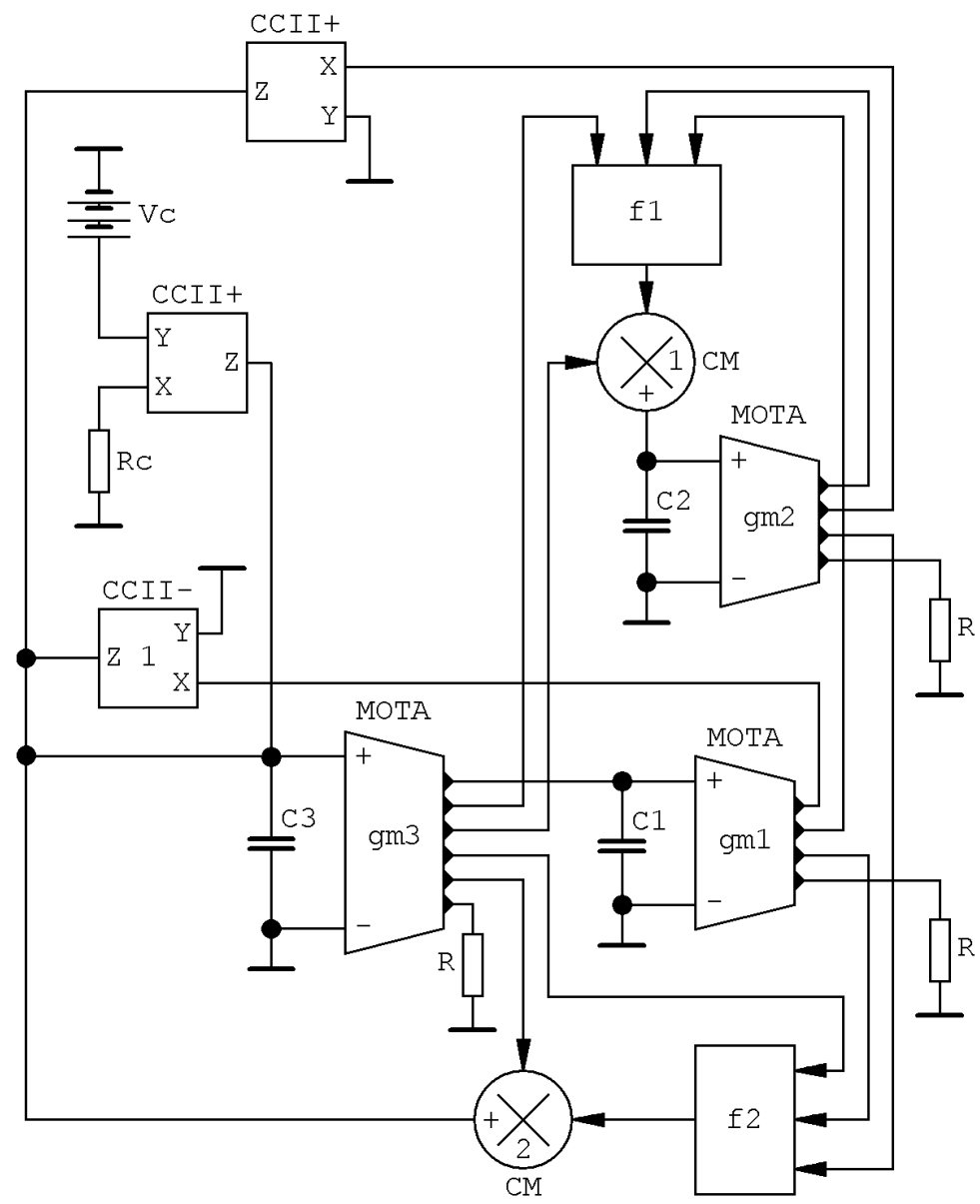

Fig. 13. Current-mode implementation of a general dynamical system having line equilibrium expressed as (2) using three MOTA, three CCII \pm and two black-boxes with desired current-mode transfer characteristics.

Circuit conception of a dynamical system (3) is illustrated in Fig. 14 and the following state equations can be derived leading to canonical realization with line equilibrium

$$
\begin{aligned}
\frac{d i_{x}}{d t} & =\frac{1}{C_{1} R_{1}} \cdot i_{z}, \quad \frac{d i_{y}}{d t}=\frac{1}{C_{2} R_{2}} \cdot \varepsilon_{1} \cdot i_{z} \cdot f_{1}\left(i_{x}, i_{y}, i_{z}\right), \\
\frac{d i_{z}}{d t} & =\frac{1}{C_{3} R_{3} R_{c}}\left[R_{x} \cdot i_{x}-R_{y} \cdot i_{y}+V_{c}\right],
\end{aligned}
$$

where the gain factors of CCII- are set to be unity. Obviously, a voltage source $V_{c}$ cannot be replaced by dc current source and designed chaotic oscillator contains only one black-box with a prescribed nonlinear current transfer function. Note that 


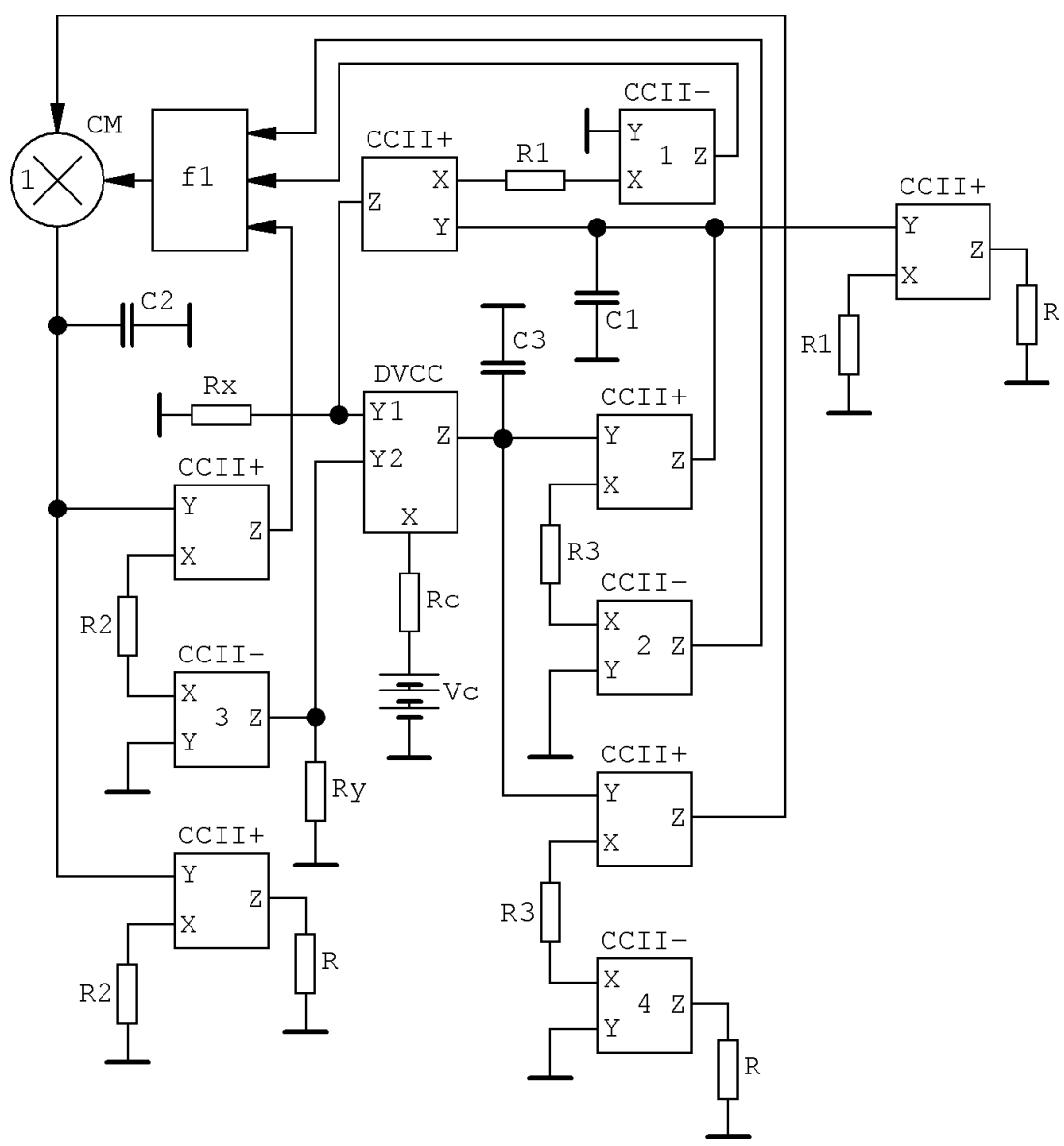

Fig. 14. Canonical current-mode realization of a dynamical system (15) using six CCII +, four CCII- and single DVCC; two-port with transfer function f1 can be arbitrary still preserving the line equilibrium.

MOCCII + is realized by four CCII \pm and whole circuit can be directly constructed and experimentally verified.

Mathematical models provided in Ref. 45 have slightly different circuit representations; for clarification, compare formulas (5), structure given in Fig. 15 and the state equations in the upcoming form

$$
\begin{aligned}
& \frac{d i_{x}}{d t}=\frac{1}{C_{1} R_{1}} \cdot i_{y}, \quad \frac{d i_{y}}{d t}=\frac{1}{C_{2} R_{2}}\left[-\gamma_{1} \cdot i_{x}+\varepsilon_{1} \cdot i_{y} \cdot i_{z}\right], \\
& \frac{d i_{z}}{d t}=\frac{-1}{C_{3} R_{3}} \varepsilon_{2} \cdot i_{x}\left(\gamma_{2} \cdot i_{y}+\gamma_{3} \cdot i_{z}+\frac{V_{c}}{R_{c}}\right) .
\end{aligned}
$$

During simulations, the circuit parameters were set to the following constant values:

$$
\gamma_{1}=\gamma_{3}=1, \quad \gamma_{2}=15, \quad \varepsilon_{1}=\varepsilon_{2}=1, \quad V_{c}=1, \quad R_{c}=10 \mathrm{k} \Omega
$$




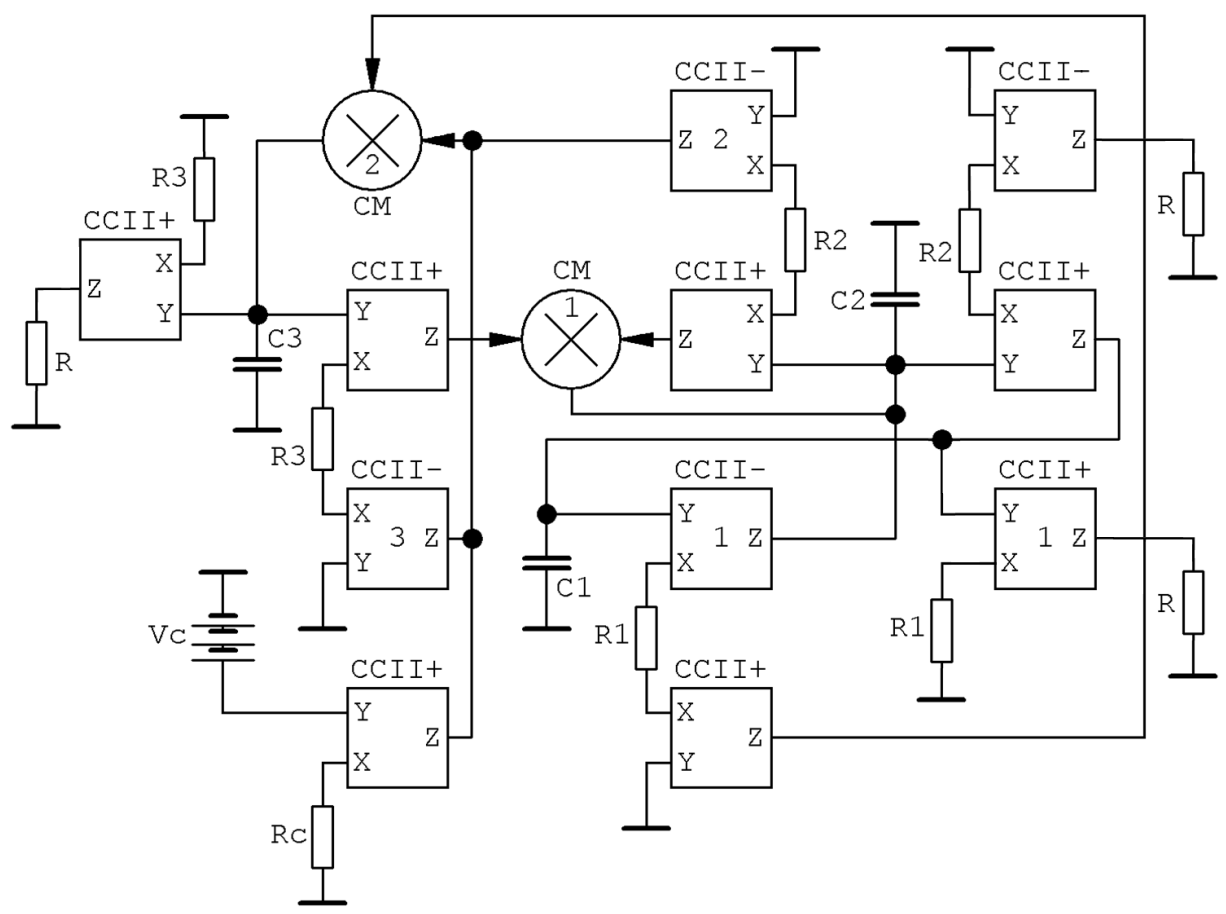

Fig. 15. Current conveyor-based current-mode network topology described by set of differential equations (16).

From an experimental perspective, these values should not represent a realization problem. Individual state variables are, as in previous cases, currents flowing through resistors $R$. This complete analog chaotic oscillator requires seven CCII+, four CCIIand two four-quadrant current multipliers. Remember that current gain 15 needs to be implemented by a cascading additional four CCII-.

By taking the concrete form of a dynamical system (4) network provided in Fig. 16 can be constructed where only three MOTA, a couple of CCII- and a pair of the current-mode multipliers are utilized. The corresponding state equations are as follows:

$$
\begin{aligned}
& \frac{d i_{x}}{d t}=\frac{g_{m 1}}{C_{1}} \cdot i_{y}+\frac{1}{R_{\mathrm{in}}^{1} C_{1}} i_{x}, \quad \frac{d i_{y}}{d t}=\frac{g_{m 2}}{C_{2}}\left[-i_{x}+\varepsilon_{1} \cdot i_{y} \cdot i_{z}\right]+\frac{1}{R_{\mathrm{in}}^{2} C_{2}} i_{x}, \\
& \frac{d i_{z}}{d t}=\frac{g_{m 3}}{C_{3}}\left[-\varepsilon_{2} \cdot i_{x}\left(\gamma_{1} \cdot i_{y}+\gamma_{2} \cdot i_{z}\right)-i_{y}\right]+\frac{1}{R_{\text {in }}^{3} C_{3}} i_{z}
\end{aligned}
$$

where $R_{\text {in }}^{k}$ represents input resistance of $k$ th OTA (for upcoming analysis of the parasitic features). During simulations, the circuit parameters were set to the following constant values:

$$
g_{m 1}=g_{m 2}=g_{m 3}=100 \mu \mathrm{S}, \quad \gamma_{1}=17, \quad \gamma_{2}=1, \quad \varepsilon_{1}=\varepsilon_{2}=1 .
$$




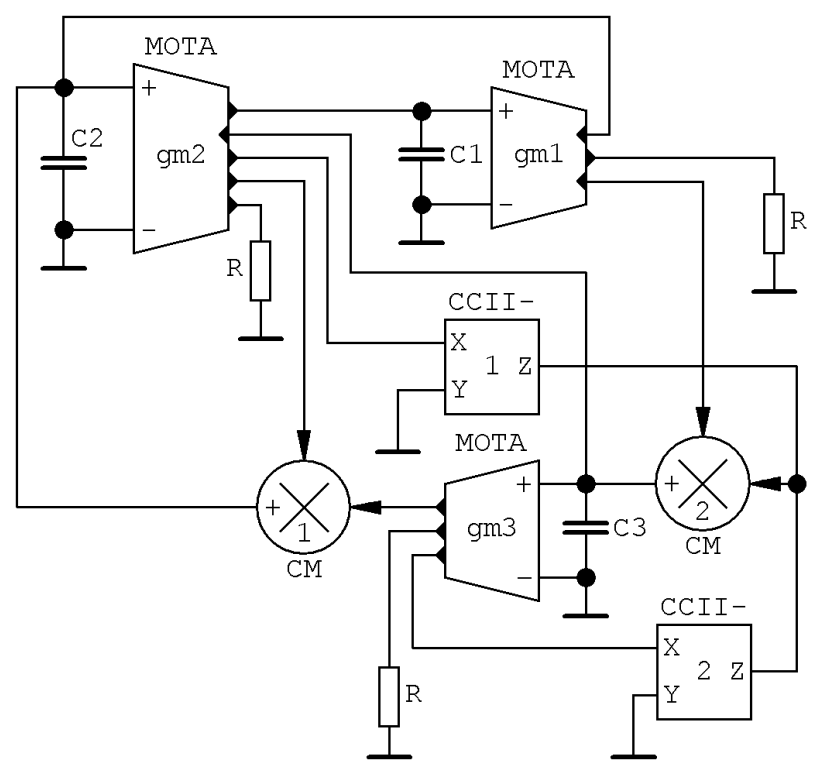

Fig. 16. OTA-based representation of a second chaotic dynamical system taken from publication. ${ }^{45}$

Since the maximum gain of EL2082 equals two, a cascade of five these active devices should be utilized to achieve desired current gain $\gamma_{1}$; in such case, total theoretical gain of 32 can be reached. Of course, in the case of on-chip implementation, high current gain is not a big problem since transfer constants of the current mirrors can be adjusted simply by the aspect ratios (W/L) of employed mosfet transistors. Be aware that CCII- as well as OTA blocks must operate in the linear regime; nonlinear part of the vector field must be generated in the prescribed form only by the current multipliers. Note that if parameter $b$ of original mathematical model is fixed to unity and only parameter $a$ is supposed to be variable second CCII- can be completely removed further simplifying final oscillator. Also, note that parasitic properties of the active elements have not been considered. In practice, their influences need to be minimized.

\subsubsection{Oscillators with conical-shaped equilibrium}

Algebraic complexity of the describing mathematical model (9a) implies that circuitry implementation will be complicated as well, see Fig. 17. This analog oscillator is covered by the following ordinary differential equations:

$$
\begin{aligned}
& \frac{d i_{x}}{d t}=\frac{-\gamma_{1}}{C_{1} R_{1}} \cdot i_{z}, \quad \frac{d i_{y}}{d t}=\frac{\varepsilon_{2}}{C_{2} R_{2}} \cdot i_{z}\left(\varepsilon_{1} \cdot i_{z}^{2}-\gamma_{5} \frac{V_{d}}{R_{d}}\right) \\
& \frac{d i_{z}}{d t}=\frac{1}{C_{3} R_{3}} \varepsilon_{5} \cdot \gamma_{2} \cdot i_{z}\left(\varepsilon_{1} \cdot i_{z}^{2}+\varepsilon_{4} \cdot \gamma_{4} \cdot i_{y}^{2}\right)+\varepsilon_{3} \cdot \gamma_{3} \cdot i_{x}^{2}-\varepsilon_{4} \cdot \gamma_{4} \cdot i_{y}^{2}-\gamma_{6} \frac{V_{c}}{R_{c}}
\end{aligned}
$$




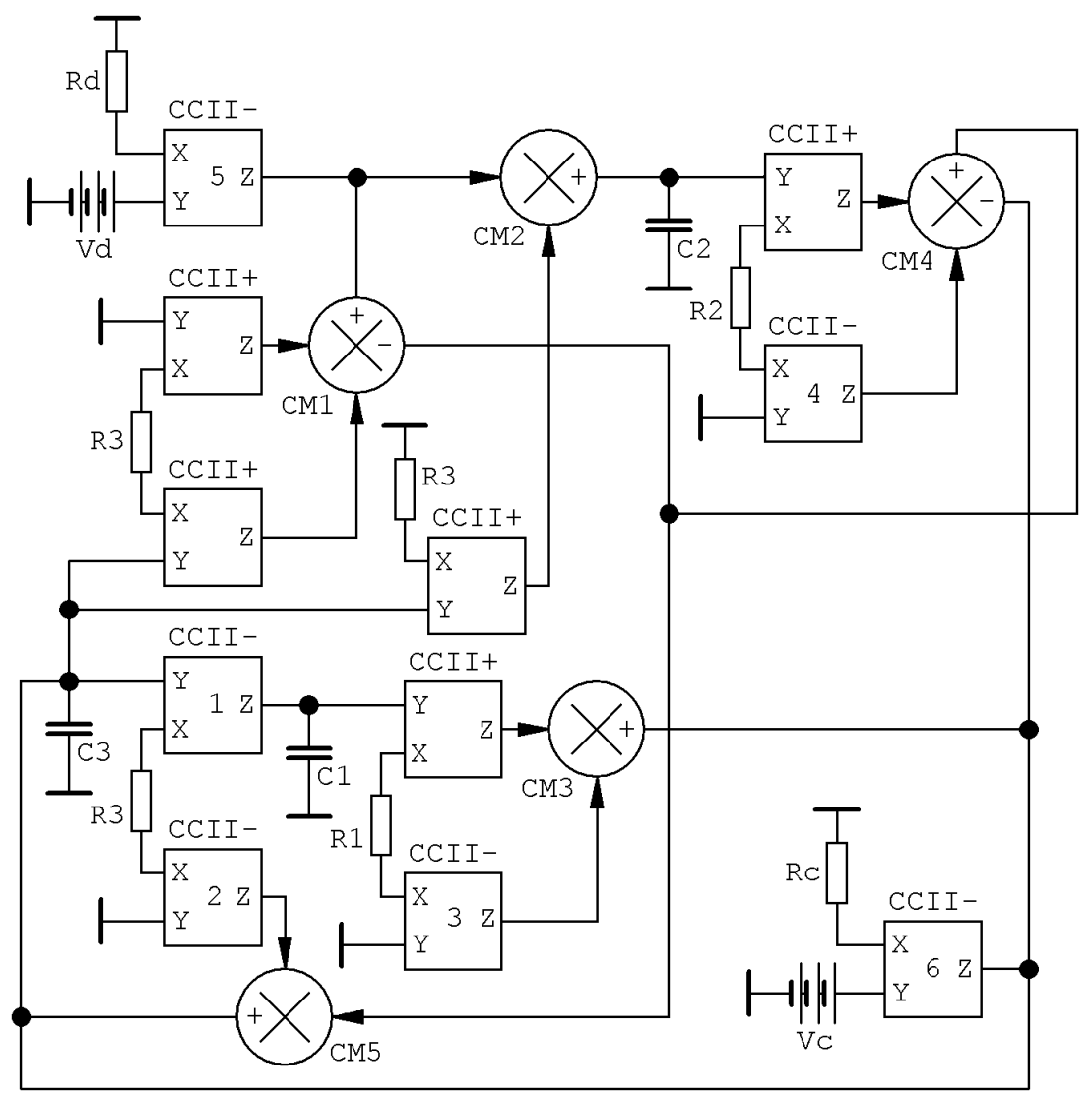

Fig. 17. Current conveyor-based current-mode network topology capable of modeling the chaotic behavior associated with dynamical system (20) having hyperbolic equilibrium, one bipolar output current multiplier is required.

where state variables can be considered as both voltages and currents, i.e., $i_{x}=u_{z} / R_{1}, i_{y}=u_{y} / R_{2}$ and $i_{z}=u_{z} / R_{3}$. External voltages $V_{c}$ and $V_{d}$ can be used as natural bifurcation parameters. Note that $11 \mathrm{CCII} \pm$ and five current multipliers are necessary for a chaotic oscillator.

Mathematical model (9b) can be realized analogically using the same building blocks as shown in Fig. 18. Set of the differential equations can be derived directly from this analog system in the form

$$
\begin{aligned}
& \frac{d i_{x}}{d t}=\frac{-\gamma_{1}}{C_{1} R_{1}} \cdot i_{z}, \quad \frac{d i_{y}}{d t}=-\frac{\varepsilon_{1} \cdot \varepsilon_{2}}{C_{2} R_{2}} \cdot i_{z}^{3}, \\
& \frac{d i_{z}}{d t}=\frac{1}{C_{3} R_{3}} \varepsilon_{4} \cdot \varepsilon_{5} \cdot \gamma_{2} \cdot i_{x} \cdot i_{y} \cdot i_{z}+\varepsilon_{3} \cdot \gamma_{3} \cdot i_{x}^{2}+\gamma_{4} \cdot i_{y}+\varepsilon_{1} \cdot i_{z}^{2},
\end{aligned}
$$




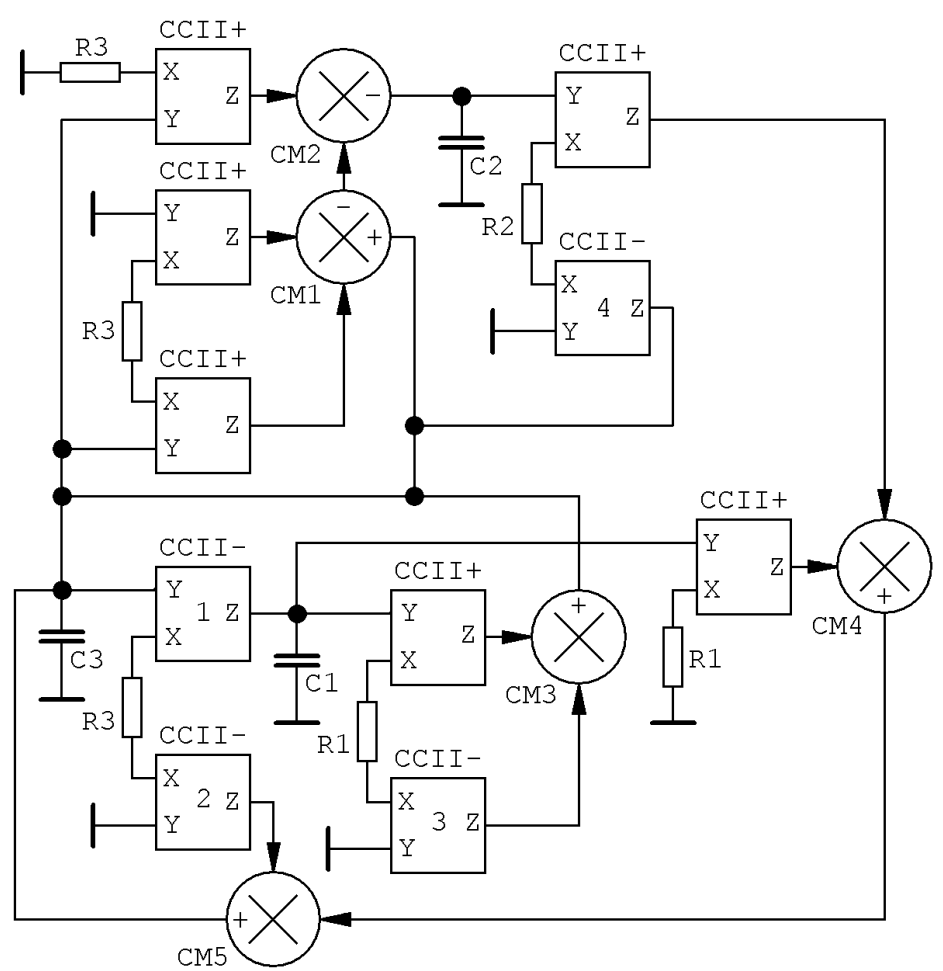

Fig. 18. Current conveyor-based current-mode chaotic circuit capable of modeling the behavior associated with system (21) having parabolic equilibrium, single bipolar output current multiplier is need.

where external voltages $V_{c}$ and $V_{d}$ can be used as suitable bifurcation parameters. Typical strange attractor can be observed, for example, for the choice

$$
\begin{aligned}
& R_{1}=R_{2}=R_{3}=R_{r}=10 \mathrm{k} \Omega, \quad V_{r}=1 \mathrm{~V}, \quad \gamma_{1}=0.1, \quad \gamma_{2}=0.03, \\
& \gamma_{3}=0.5, \quad \gamma_{4}=2, \quad \varepsilon_{1}=0.35, \quad \varepsilon_{2}=12.5, \quad \varepsilon_{3}=\varepsilon_{4}=\varepsilon_{5}=1 .
\end{aligned}
$$

Note that the proposed collection of values should not represent serious realization problems. If directly unreachable constant $\varepsilon_{2}$ can be lowered to 1.25 while $R_{2}$ changes to $1 \mathrm{k} \Omega$.

\subsubsection{Electronic system with elliptical-type equilibrium}

Similarly, describing state equations of autonomous analog network capable of modeling the dynamical system with circular equilibrium are as follows:

$$
\begin{aligned}
& \frac{d i_{x}}{d t}=\frac{1}{C_{1} R_{1}} \cdot i_{z}, \quad \frac{d i_{y}}{d t}=\frac{1}{C_{2} R_{2}} \varepsilon_{1} \cdot i_{z}\left(i_{x}+\varepsilon_{2} \cdot i_{z}^{2}\right), \\
& \frac{d i_{z}}{d t}=\frac{1}{C_{3} R_{3}}\left[\varepsilon_{3} \cdot i_{x} \cdot i_{z}+\varepsilon_{4} \cdot i_{x}^{2}+\varepsilon_{5} \cdot i_{y}^{2}-\gamma_{1} \frac{V_{r}}{R_{r}}\right],
\end{aligned}
$$




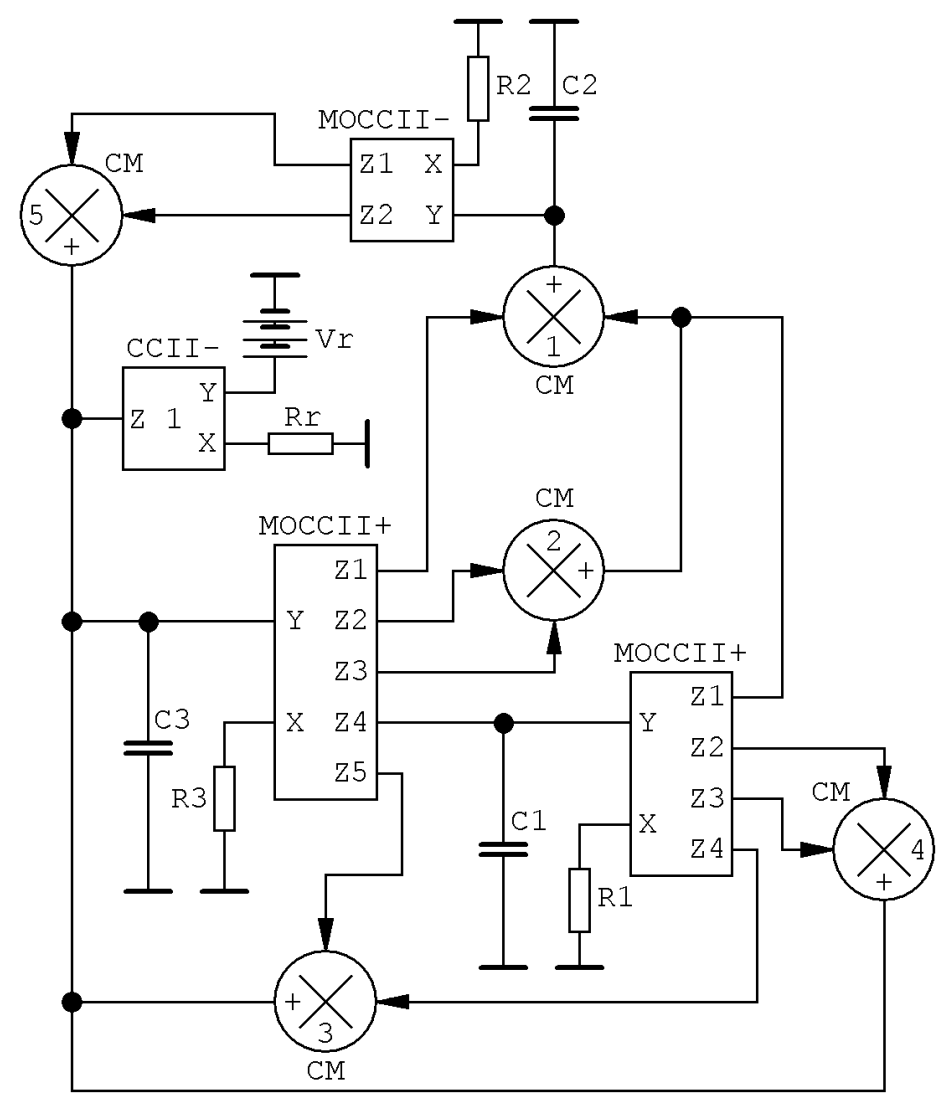

Fig. 19. Current-mode circuit realization of a system (23) with circular equilibrium using MOCCII+ elements.

where circle radius can be changed directly by the external voltage $V_{r}$ or, more precisely, by transfer gain $\gamma_{1}$. Careful adjustment is required here since global behavior is extremely sensitive to this value. Corresponding network can be found in Fig. 19. Unfortunately, MOCCII+ is not off-the-shelf active component; thus, the previous design cannot be used for immediate laboratory experimentation and transformation into CCII \pm only based network is necessary. After a slight modification, a chaotic oscillator shown in Fig. 20 has been achieved. This circuit is covered by a set of the ordinary differential equations

$$
\begin{aligned}
& \frac{d i_{x}}{d t}=\frac{g_{m 1}}{C_{1}} \cdot i_{z}, \quad \frac{d i_{y}}{d t}=\frac{g_{m 2}}{C_{2}}\left(-\varepsilon_{1} \frac{R_{y}}{R_{x}} i_{x} \cdot i_{z}-\varepsilon_{2} \cdot \varepsilon_{5} \cdot i_{z}^{3}\right), \\
& \frac{d i_{z}}{d t}=\frac{g_{m 3}}{C_{3}}\left(\varepsilon_{1} \frac{R_{y}}{R_{x}} i_{x} \cdot i_{z}+\varepsilon_{3} \cdot i_{x}^{2}+\varepsilon_{4} \cdot i_{y}^{2}-\frac{V_{r}}{R_{r}}\right) .
\end{aligned}
$$




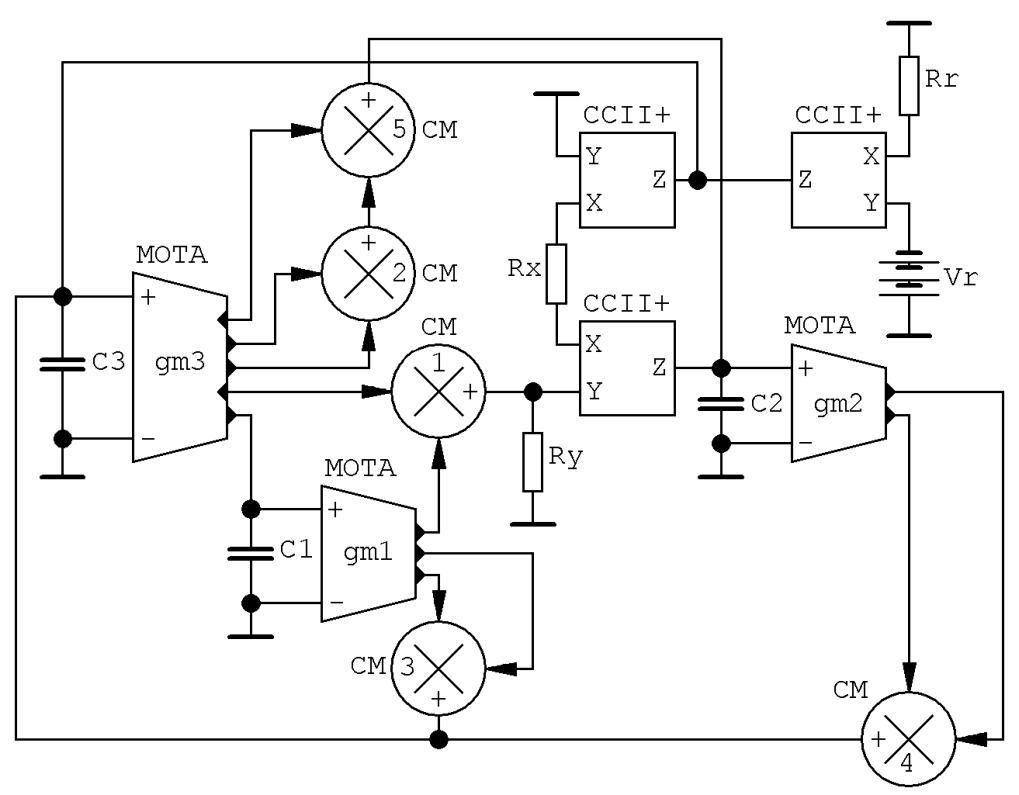

Fig. 20. Current-mode circuit realization of a dynamical system (24) with circular equilibrium structure.

For computer-aided verification, most circuits can be fixed on circuit-reasonable numerical values such as

$$
\begin{aligned}
& g_{m 1}=10 \mu \mathrm{S}, \quad g_{m 2}=4 \mathrm{mS}, \quad g_{m 3}=100 \mu \mathrm{S}, \quad R_{y}=R_{r}=1 \mathrm{k} \Omega, \\
& R_{x}=10 \mathrm{k} \Omega, \quad \varepsilon_{1}=\varepsilon_{3}=\varepsilon_{4}=1, \quad \varepsilon_{2}=0.1, \quad \varepsilon_{5}=0.8, \quad V_{r}=1 \mathrm{~V} .
\end{aligned}
$$

For these values, the ratio between minor/major axes of ellipse $r_{1} / r_{2}$ is enhanced to $1 / 3$.

In any case, only a fraction of ellipse provides a vector field geometry responsible for the evolution of this strange attractor. However, such fraction still represents infinite number of the equilibrium points. Major and minor radius of an equilibrium ellipse equals inverse square root of current gain factors $\varepsilon_{3}$ and $\varepsilon_{4}$, respectively. Numerical studies reveal that the global behavior of this dynamical system is extremely sensitive to both radiuses and chaos quickly disappears for values far away from unity; a solution became unbounded leading to the state space attractor limited only by the saturation levels of the used active devices (in fact applied supply voltages).

Note that only transfer factors of the used current multipliers are supposed to control chaotic motion. Since EL4083 is the only commercially available current multiplier so far (but without chance to adjust gain), this circuit should be considered for on-chip CMOS realization and fabrication. Extreme sensitivities of a specific strange attractor to external currents can turn to be advantageous if these circuit quantities are generated by some sort of sensors, for example, caused by the chemical changes in some liquid. 
Canonical dynamical system having elliptical equilibrium can also be implemented in various current-mode conceptions. One of them can be described by state equations

$$
\begin{aligned}
& \frac{d i_{x}}{d t}=\frac{g_{m 1}}{C_{1}} \cdot i_{z}, \quad \frac{d i_{y}}{d t}=\frac{g_{m 2}}{C_{2}} \varepsilon_{1} \cdot i_{z} \cdot f_{1}\left(i_{x}, i_{y}, i_{z}\right), \\
& \frac{d i_{z}}{d t}=\frac{g_{m 3}}{C_{3}}\left(\gamma_{1} \cdot \varepsilon_{2} \cdot i_{x}^{2}+\gamma_{2} \cdot \varepsilon_{3} \cdot i_{y}^{2}-\frac{V_{r}}{R_{r}}\right),
\end{aligned}
$$

where $V_{r}=1 \mathrm{~V}$ is fixed constant and value $R_{r}$ should be chosen according to impedance normalization factor. Major and minor radius of an equilibrium ellipse equals inverse square root of current gain factors $\varepsilon_{2}$ and $\varepsilon_{3}$, respectively. This so far unfinished analog circuit where two-port with arbitrary transfer function is given as black-box is provided by means of Fig. 21.

Finally, network structure with only CCII \pm and current multipliers can be derived as shown in Fig. 22. Straightforward analysis leads to the following describing formulas:

$$
\begin{aligned}
& \frac{d i_{x}}{d t}=\frac{-\gamma_{1}}{C_{1} R_{1}} \cdot i_{z}, \quad \frac{d i_{y}}{d t}=\frac{\varepsilon_{2} \cdot i_{z}}{C_{2} R_{2}}\left(\varepsilon_{1} \cdot i_{z}^{2}+i_{x}\right) \\
& \frac{d i_{z}}{d t}=\frac{1}{C_{3} R_{3}}\left(\varepsilon_{2} \cdot \varepsilon_{5} \cdot \gamma_{2} \cdot i_{x} \cdot i_{z}+\varepsilon_{4} \cdot \gamma_{4} \cdot i_{x}^{2}+\varepsilon_{3} \cdot \gamma_{3} \cdot i_{y}^{2}+\frac{V_{r}}{R_{r}}\right) .
\end{aligned}
$$

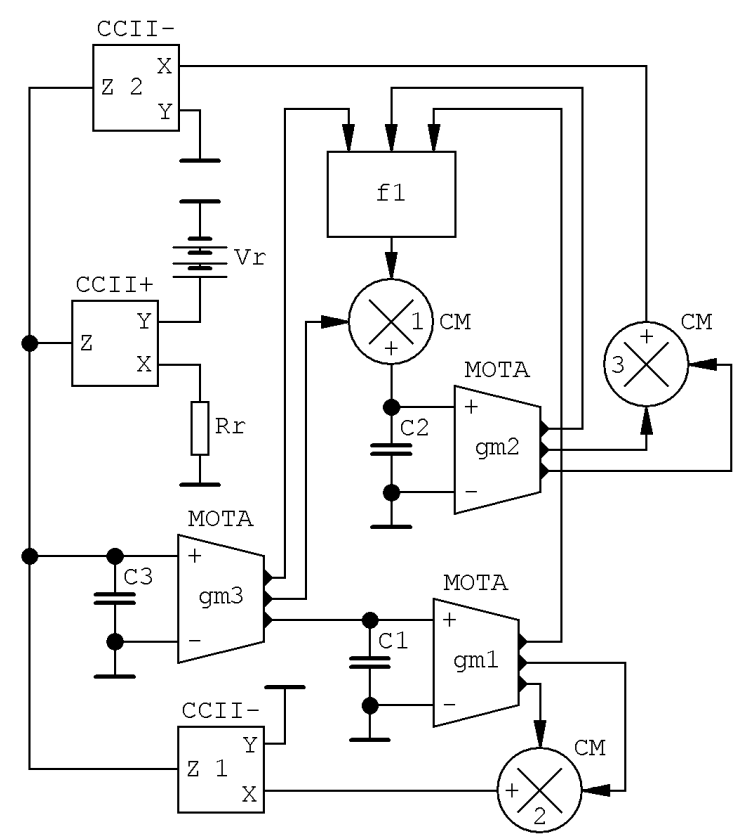

Fig. 21. General concept of a chaotic oscillator with elliptical equilibrium where function f1 can be arbitrary. 


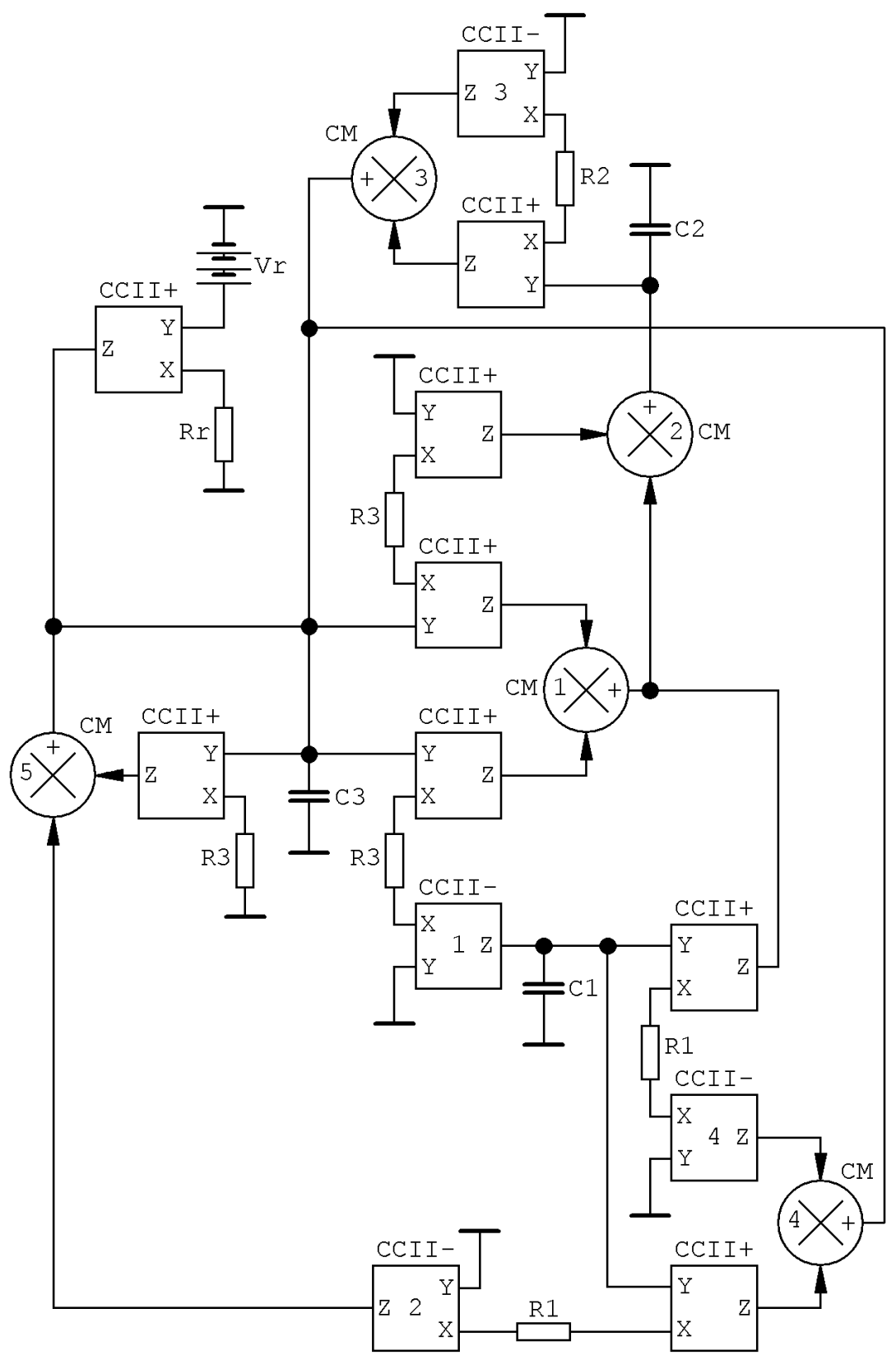

Fig. 22. Fully current-mode chaotic oscillator with elliptical equilibrium ready for experimental measurement; design requirements are eight $\mathrm{CCII}+$, four CCII- and five current-mode multipliers. 
Numerical values of the circuit parameters leading to the evolution of a typical strange attractor can be chosen as follows:

$$
\begin{aligned}
& R_{1}=R_{2}=R_{3}=R_{r}=10 \mathrm{k} \Omega, \quad \gamma_{1}=0.07, \quad \gamma_{2}=1, \quad V_{r}=1 \mathrm{~V}, \\
& \varepsilon_{1}=0.5, \quad \varepsilon_{2}=4, \quad \varepsilon_{3}=0.66, \quad \varepsilon_{4}=1.52, \quad \varepsilon_{5}=0.1 .
\end{aligned}
$$

\subsection{Orcad Pspice circuit simulations}

Orcad Pspice simulator has been utilized for demonstrating that chaotic solution is neither a numerical artifact nor a transient motion but a regular solution. To preserve a limited length of this paper, only strange attractors are visualized. These are in very good accordance with the theoretical expectations, i.e., with the numerically integrated state trajectories. Since chaotic oscillators are always nonlinear circuits, simulation scenarios are restricted to time-domain analysis; final time was chosen to be $100 \mathrm{~ms}$ and maximum step size $1 \mu \mathrm{s}$ with respect to a time constant. Achieved waveforms are sufficiently smooth and long enough such that the calculated frequency spectra of the generated signals (using fast Fourier transform) have the required resolution.

First simulation results given in this section are related to the dynamical system with line equilibrium (16) with values (17). Chaotic waveforms in time and frequency

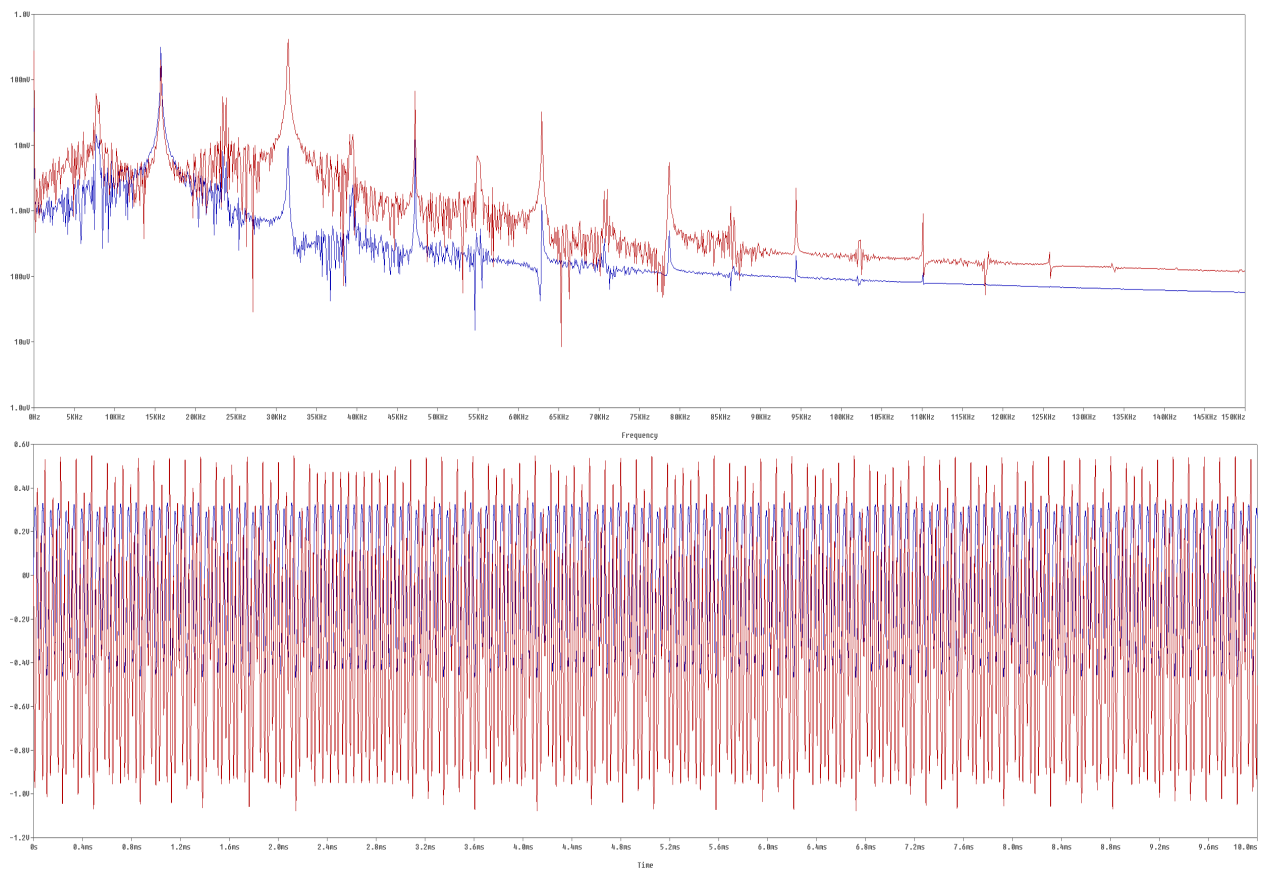

Fig. 23. Chaotic signals in time domain (lower picture) and calculated frequency components (upper picture). 


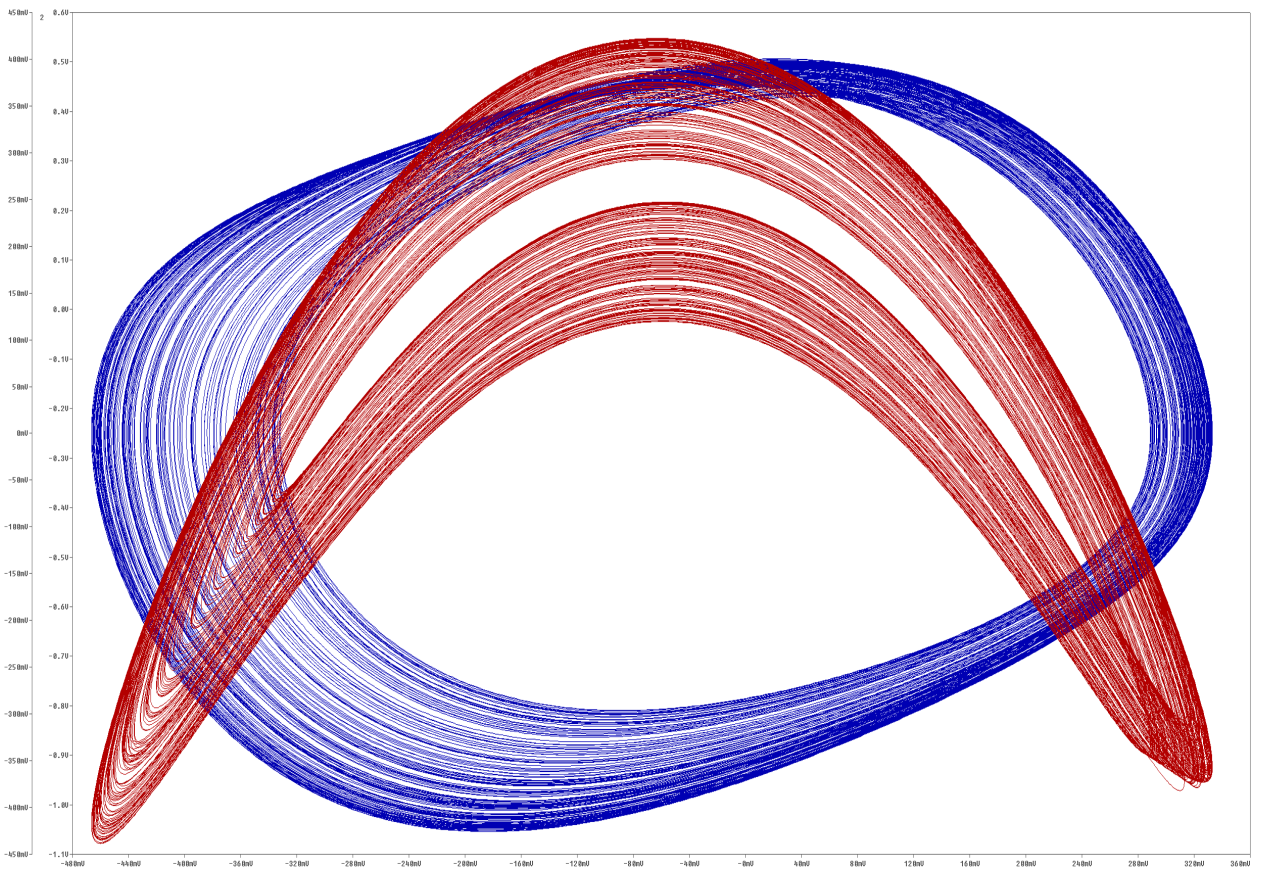

Fig. 24. Plane projections $i_{y}$ and $i_{z}$ versus $i_{x}$ of a typical chaotic attractor generated by analog circuit given in Fig. 15, described by differential equations (16) with numerical values (17).

domain are provided in Fig. 23 (up to frequency component $150 \mathrm{kHz}$ ) and selected plane projections of a typical strange attractor are shown in Fig. 24.

Second simulation results are bounded to a dynamical system with a line equilibrium, namely, which is described by the differential equations (18) together with the numerical values (19). Generated chaotic signals in time and frequency domain are shown by means of Fig. 25 while corresponding plane projections of a state attractor are given in Fig. 26. The state variables are naturally currents, but for attractor visualization purpose, voltages across grounded capacitors are considered. Note that the chaotic waveforms generated by this dynamical system have several dominant peaks in the frequency domain.

Third circuit which was verified by simulation is specified by the equations (24) with the numerical values (25). Resulting chaotic waveforms plotted in time and frequency domain (visualization provided up to $2 \mathrm{kHz}$ ) are shown in Fig. 27. Corresponding Monge projections can be found in Fig. 28.

Fourth analog chaotic oscillator which undergoes verification through simulation is given by the differential equations (27) together with a parameter choice (28). Generated chaotic signals in time and frequency domain (wideband linear-scaled axis $0 \mathrm{kHz}$ to $200 \mathrm{kHz}$ is visualized) are shown in Fig. 29. Same waveforms plotted as the plane projections are provided by means of Fig. 30. 


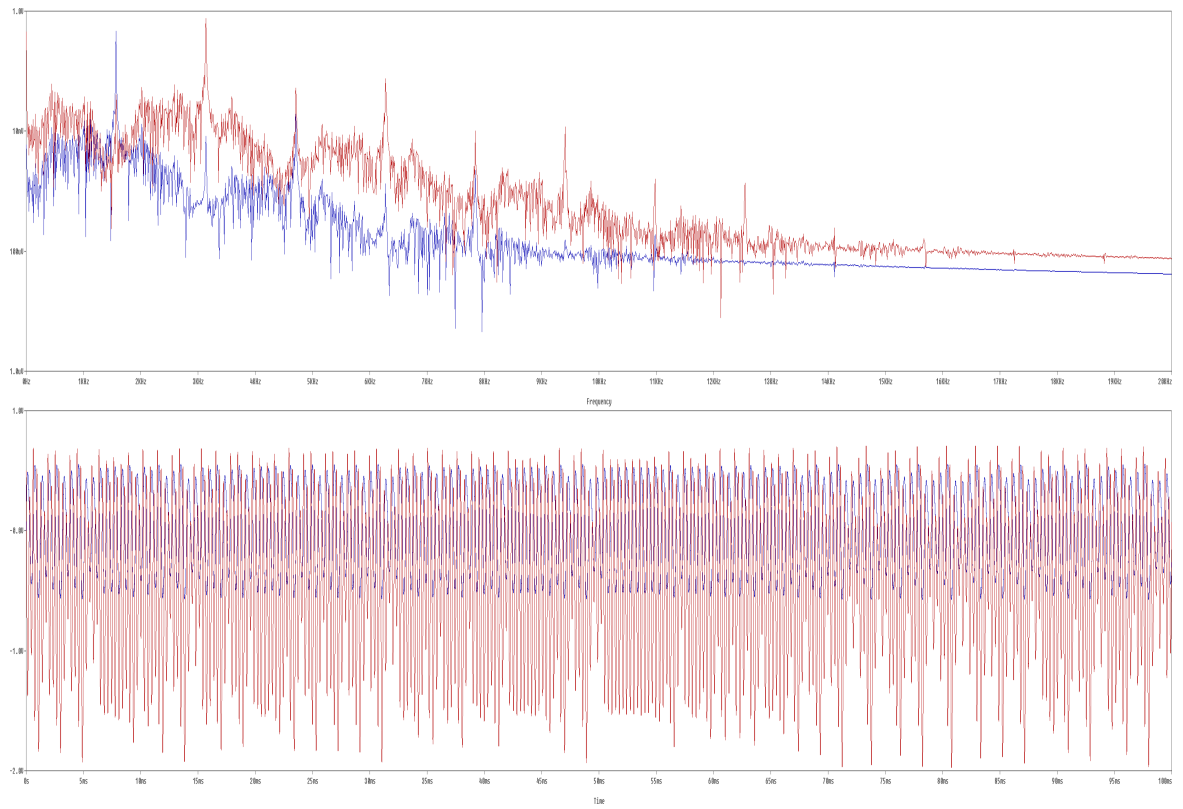

Fig. 25. Chaotic signals in time domain (lower picture) and calculated frequency components (upper picture).

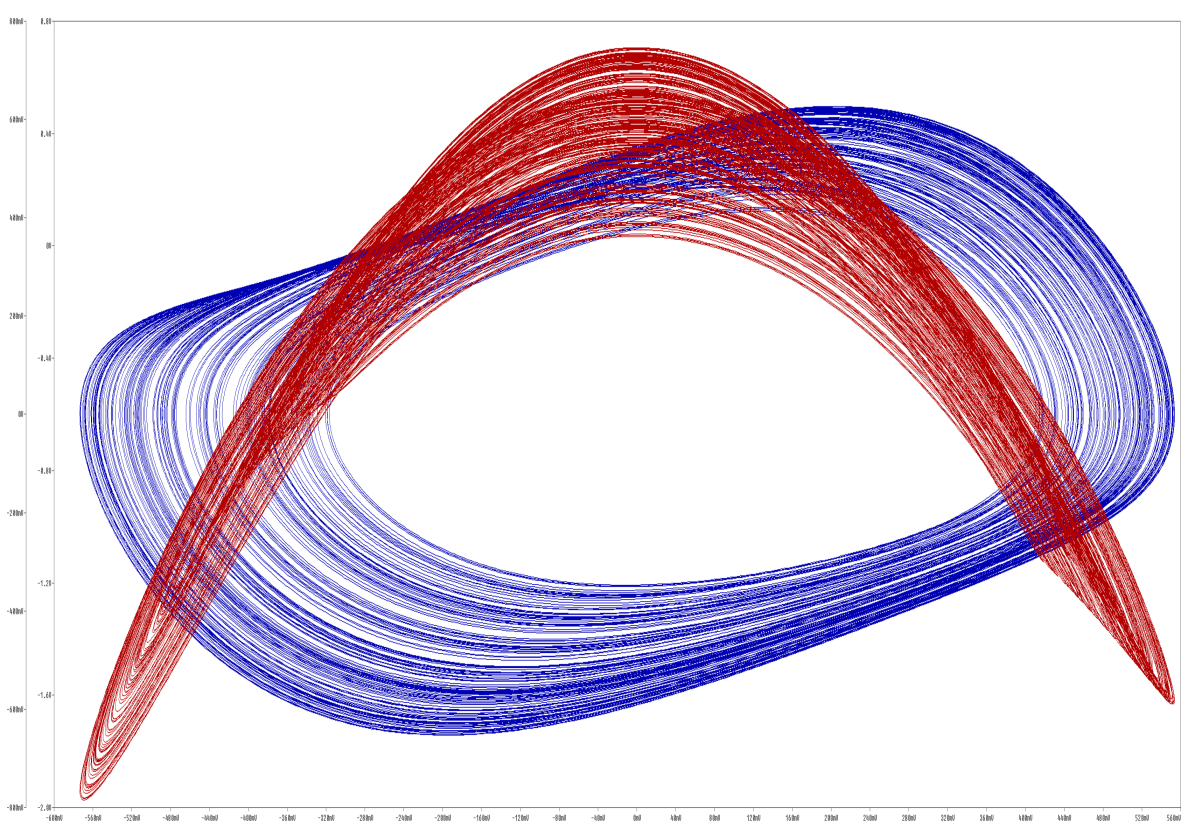

Fig. 26. Plane projections $i_{y}$ and $i_{z}$ versus $i_{x}$ of a typical chaotic attractor generated by circuit provided in Fig. 16, described by the differential equations (18) with numerical values (19). 

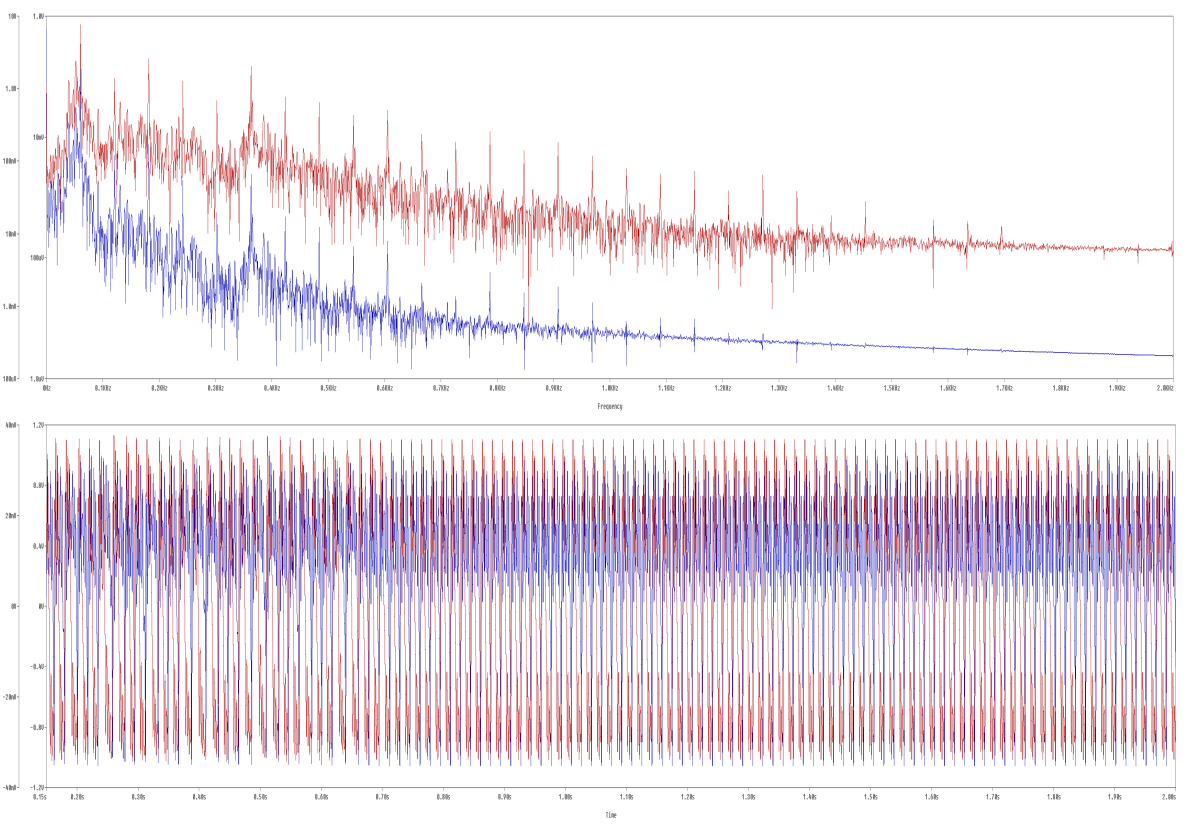

Fig. 27. Chaotic signals in time domain (lower picture) and calculated frequency components (upper picture).

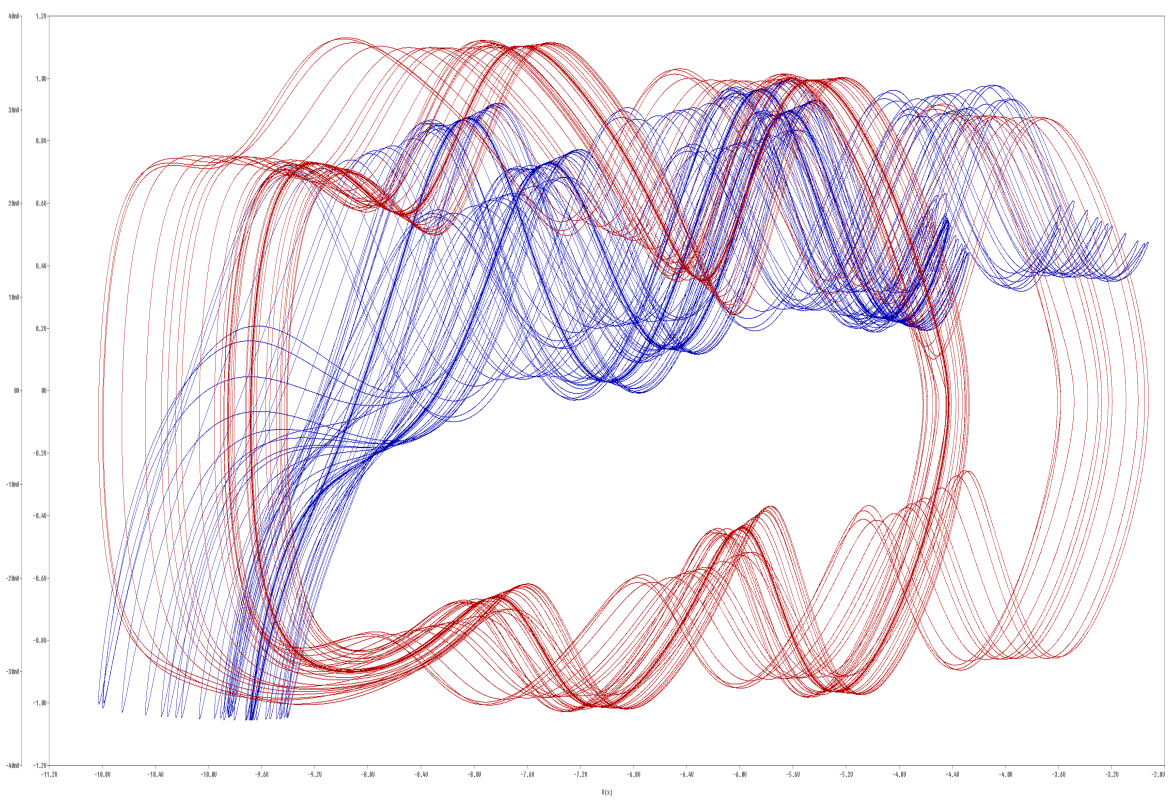

Fig. 28. Plane projections $i_{y}$ and $i_{z}$ versus $i_{x}$ of a typical chaotic attractor generated by circuit given in Fig. 20, described by the differential equation (24) with numerical values (25). 

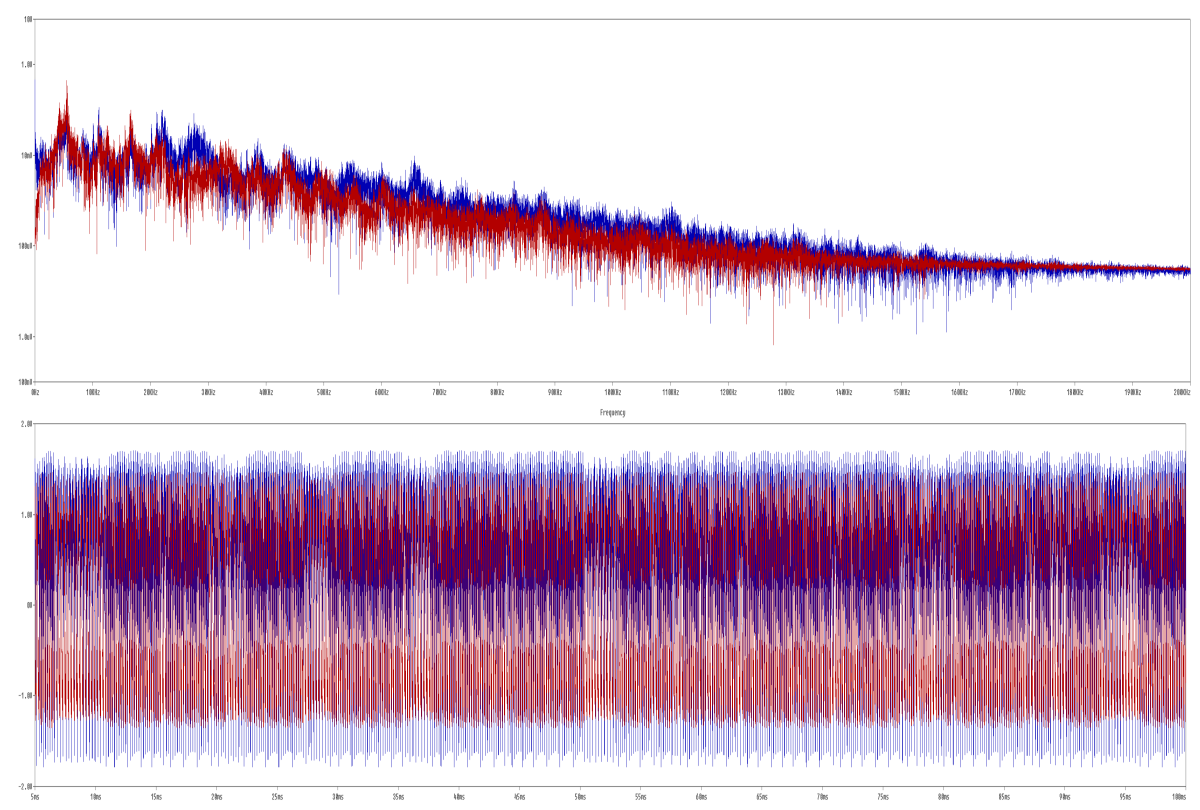

Fig. 29. Chaotic signals in time domain (lower picture) and calculated frequency components (upper picture).

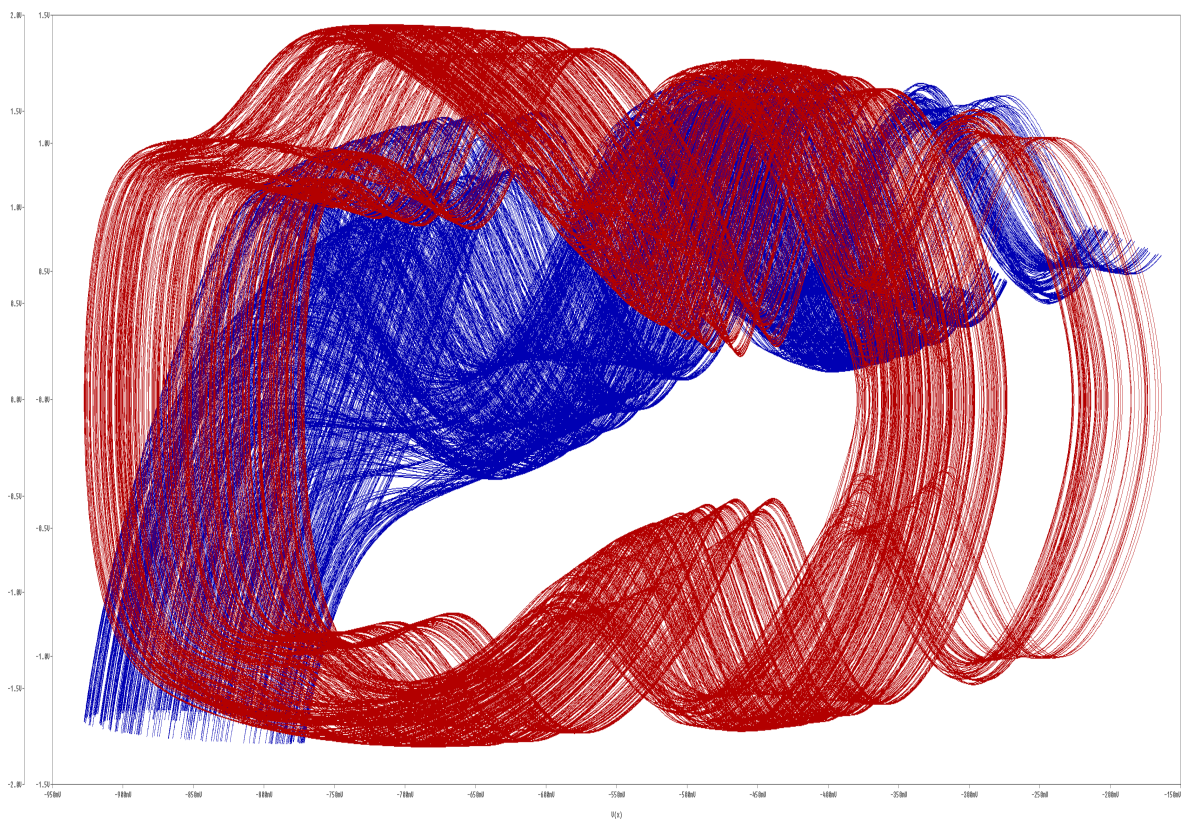

Fig. 30. Monge projections $i_{y}$ and $i_{z}$ versus $i_{x}$ of a typical chaotic attractor generated by the analog circuitry depicted in Fig. 22, described by the differential equations (27) using numerical values (28). 


\section{Concept of Generalized Parasitic and Its Analysis}

Fast dynamical motions and short transients can be found to be quite problematic in the case of circuit implementation of prescribed dynamics. Mathematically speaking, such situation corresponds to a right-hand side of the differential equations multiplied by a big number. If we consider parasitic properties of the used active elements, error terms will necessarily appear in a set of describing differential equations. This can also cause nasty phenomena such as significant increase of a network order, reducing degrees of freedom by bounding two accumulation elements, it can lead to a structural instability of a chaotic attractor or completely damage the prescribed state attractor. Since chaotic orbit is typically surrounded in hyperspace of the internal system parameters by unbounded solution (i.e., solution going to \pm infinity), this attractor collapses into a large limit cycle with a squared quasi-radius defined by the saturation levels of used active devices (these ranges are slightly reduced supply voltages). Deep inspection of published papers dealing with continuous-time chaotic oscillator design reveals that authors usually care little about the parasitic properties of used active elements and how these factors influence expected strange attractor. Of course, there are a few exceptions like ${ }^{75}$ where authors nicely explain how frequency limitations of AD844 affect construction and verification of the multi-scroll chaotic oscillator. Anyhow, theoretically such qualitative analysis can solve this problem: Is desired chaotic pattern structurally stable such that the generated waveforms have the potential for the practical applications? If such stability cannot be satisfied to some degree, desired chaotic attractor is not experimentally observable. The major problem here is that neither largest LE nor geometrical dimension is a monotonic function with respect to the parasitic element values. Considering this, it means that common worst-case analysis or systematic gradient optimization methods do not represent the correct approach to determine the structural stability of the state space attractors in the case of the nonlinear vector field.

Let us imagine that parasitic properties of the individual active devices are considered separately. Each such parameter has unknown value and forms one edge of hyperspace scanned by optimization routine. This idea is wrong both from viewpoint of visualization and enormous time demands required for calculation. To quantify influence of nonideal properties of the active devices on the desired strange attractors a new term generalized parasitic can be defined. It means that parasitic effects which have the same nature are swept and applied on mathematical model of chaotic dynamical system together in group. The most important generalized parasitic effect is additional dissipation (caused by finite input resistances of CCII \pm , OTA, DVCC blocks or output resistances of these elements), parameter uncertainty, roll-offs (caused by low-pass nature of $\mathrm{CCII} \pm$ or OTA transfers) and component tolerances. An example of how to deal with the mentioned reality is provided in Fig. 31 where generalized parasitic analysis is put into the context with the so-called 


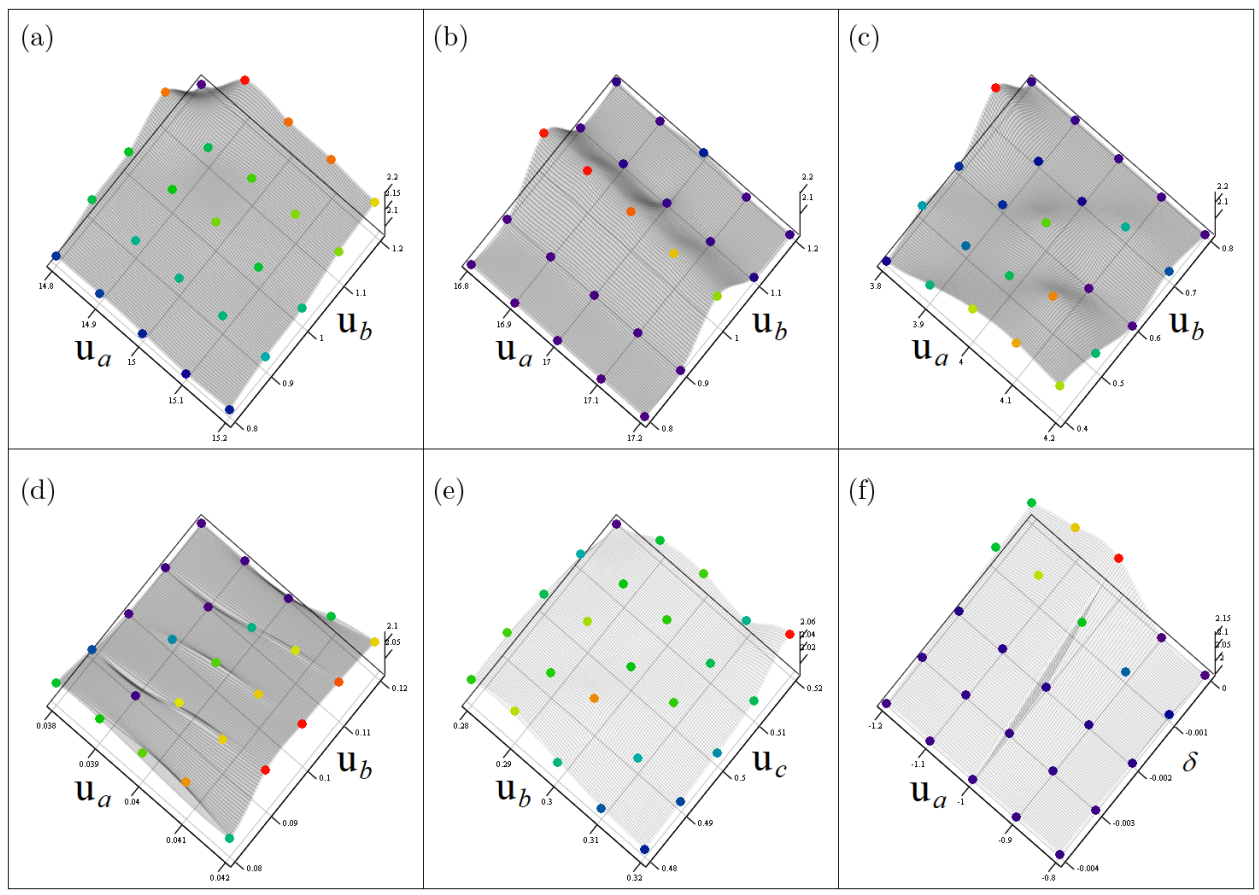

Fig. 31. Graphical illustration of a generalized parasitic concept for a system (2) with functions: (a) choice (5a), (b) choice (5b), (c) set (5d), (d) set (5e), (e) set (7), (f) uncertainty of parameter $a$ and additional dissipation for $(9 \mathrm{a})$.

Kaplan-Yorke dimension of the observed state attractor. In this plot, colored points are rainbow-scaled such that red denotes strongly chaotic attractor, yellow represents weakly chaotic system, green and blue stand for limit cycles. Horizontal grid is sparse such that it is easy to recognize nominal (ideal) system state. This idea is applied on the selected state equations. Note that this method is capable of finding "more chaotic" systems.

Since one basic error term never compensates the other, analyzed parasitic properties tend to have an accumulating nature. For example, each OTA block connected to a working capacitor enlarges time constant by associated parasitic input capacitance while its input resistance is responsible for increased dissipation of dynamical flow. If dissipation is too high (above critical value), desired strange attractor collapses into the simpler geometrical structure, i.e., limit cycle or fixed point. In CCII-based chaotic oscillators, input resistance of $X$-terminal is connected in series with working resistor causing again a time constant enlargement effect. Roll-off effect of each OTA transconductance as well as each CCII current transfer constant (both should be ideally constant over full frequency scale) also has a devastating impact on a desired state attractor. 


\section{Conclusions}

The first part of this contribution can be considered as a comprehensive review showing the process of discovering mathematical models with exotic types and shapes of equilibrium, beginning with the simplest dissipative flows ${ }^{82}$ to the hyperchaotic systems with 3D equilibrium structures or a chaotic dynamic where the attractor can be changed from hidden to self-excited ${ }^{83}$ by changing the values of the internal system parameters.

The main part of this paper is focused on current-mode circuit realizations and consequent simulations of the hidden chaotic oscillators with the degenerated equilibrium structures. It completes current research studies where voltage-mode operational regime is preferred over current-mode designs. It is also for the first time when fully current-mode circuitry realization of a dynamical system with circular and elliptical equilibrium is reported, although the existence of a desired strange attractor is proved only by Orcad Pspice circuit simulations using datasheet-based level-three models of the active devices.

Last idea behind this paper is the suggestion to adopt a concept of 1D LE (used for calculation of a metric dimension of state attractor) for case-specific sensitivity analysis: to determine the robustness of the designed chaotic oscillator to preserve prescribed strange attractor. The most devastating parasitic properties of utilized active elements are specified, and the proposed procedure is demonstrated via example.

\section{Acknowledgments}

Research described in this paper was financed by the National Sustainability Program under grant LO1401 and the Czech Science Foundation under grant number GA15-22712S. For the research, infrastructure of the SIX Center was used.

\section{References}

1. J. C. Sprott, A new class of chaotic circuit, Phys. Lett. A 266 (2000) 19-23.

2. J. R. Piper and J. C. Sprott, Simple autonomous chaotic circuits, IEEE Trans. Circuits Syst. II, Express Briefs 57 (2010) 730-734.

3. J. C. Sprott, A new chaotic jerk circuit, IEEE Trans. Circuits Syst. II, Express Briefs $\mathbf{5 8}$ (2011) 240-243.

4. J. Petrzela, Modeling deterministic chaos using electronic circuits, Radioengineering 20 (2011) 438-444.

5. M. Itoh, Synthesis of electronic circuits for simulating nonlinear dynamics, Int. J. Bifurcation Chaos 11 (2001) 605-653.

6. S. Yu, J. Lu, H. Leung and G. Chen, Design and implementation of n-scroll chaotic attractors from a general jerk circuit, IEEE Trans. Circuits Syst. I, Fundam. Theory Appl. 52 (2005) 1459-1476. 
7. J. E. Varrientos and E. Sanchez-Sinencio, A 4-D chaotic oscillator based on a differential hysteresis comparator, IEEE Trans. Circuits Syst. I, Fundam. Theory Appl. 45 (1998) $3-10$.

8. X. S. Yang, Q. Li and G. Chen, A twin-star hyperchaotic attractor and its circuit implementation. Int. J. Circuit Theory Appl. 31 (2003) 637-640.

9. T. Gotthans and J. Petrzela, Experimental study of the sampled labyrinth chaos, Radioengineering 20 (2011) 873-879.

10. L. Chua and G. N. Lin, Canonical realizations of Chua's circuit family, IEEE Trans. Circuits Syst. I, Fundam. Theory Appl. 37 (1990) 885-902.

11. M. Gotz, U. Feldman and W. Schwarz, Synthesis of higher dimensional Chua circuits, IEEE Trans. Circuits Syst. I, Fundam. Theory Appl. 40 (1993) 854-860.

12. W. K. S. Tang, G. Q. Zhong, G. Chen and K. F. Man, Generation of N-scroll attractors via sine function, IEEE Trans. Circuits Syst. I, Fundam. Theory Appl. 48 (2001) 13691372 .

13. S. O. Scanlan, Synthesis of piecewise-linear chaotic oscillators with prescribed eigenvalues, IEEE Trans. Circuits Syst. I, Fundam. Theory Appl. 46 (1999) 1057-1064.

14. J. Petrzela and J. Slezak, Conservative chaos generators with CCII+ based on mathematical model of nonlinear oscillator, Radioengineering 17 (2008) 19-24.

15. R. Trejo-Guerra, E. Tlelo-Cuautle, C. Cruz-Hernandez and C. Sanchez-Lopez, Chaotic communication system using Chua's oscillators realized with CCII $+\mathrm{s}$, Int. J. Bifurcation Chaos 19 (2009) 4217-4226.

16. A. S. Elwakil and S. Ozoguz, On the generation of higher order chaotic oscillators via passive coupling of two identical or nonidentical sinusoidal oscillators, IEEE Trans. Circuits Syst. I, Fundam. Theory Appl. 53 (2006) 1521-1532.

17. A. S. Elwakil and M. P. Kennedy, Construction of classes of circuit-independent chaotic oscillators using passive-only nonlinear devices, IEEE Trans. Circuits Syst. I, Fundam. Theory Appl. 48 (2001) 289-307.

18. A. S. Elwakil and A. M. Soliman, Current conveyor chaos generators, IEEE Trans. Circuits Syst. I, Fundam. Theory Appl. 46 (1999) 393-398.

19. A. S. Elwakil and M. P. Kennedy, Chaotic oscillator configuration using a frequency dependent negative resistor, Int. J. Circuit Theory Appl. 28 (2000) 67-76.

20. A. S. Elwakil and M. P. Kennedy, A family of Wien-type oscillators modified for chaos, Int. J. Circuit Theory Appl. 25 (1997) 561-579.

21. J. Petrzela, On the existence of chaos in the electronically adjustable structures of the state variable filters, Int. J. Circuit Theory Appl. 44 (2016) 1779-1797.

22. J. Petrzela, Chaotic behavior of state variable filters with saturation-type integrators, Electron. Lett. 51 (2015) 1159-1161.

23. H. Li, Ch. Li, Z. Yuan, W. Hu and X. Zhen, A new class of chaotic circuit with logic elements, J. Circuits, Systems Comput. 24 (2015) 1550136.

24. R. Trejo-Guerra, E. Tlelo-Cuautle, J. M. Jimenez-Fuentes, C. Sanchez-Lopez, J. M. Munoz-Pacheco, G. Espinosa-Flores-Verdad and J. M. Rocha-Perez, Integrated circuit generating 3- and 5-scroll attractors, Commun. Nonlinear Sci. Numer. Simul. 17 (2012) 4328-4335.

25. A. G. Radwan, A. M. Soliman and A. L. El-Sedeek, Low-voltage MOS chaotic oscillator based on the nonlinearity of gm, J. Circuits, Systems Comput. 13 (2004) 101.

26. R. Trejo-Guerra, E. Tlelo-Cuautle, J. M. Jimenez-Fuentes, J. M. Munoz-Pacheco and C. Sanchez-Lopez, Multiscroll floating gate based integrated chaotic oscillator, Int. J. Circuit Theory Appl. 41 (2013) 831-843. 
27. J. C. Nunez, E. Tlelo, C. Ramirez and J. M. Chimenez, CCII+ based on QFGMOS for implementing Chua's chaotic oscillator, IEEE Latin Am. Trans. 13 (2015) 2865-2870.

28. A. D. Pano-Azucena, J. J. Rangel-Magdaleno, E. Tlelo-Cuautle and A. J. Quintas-Valles, Arduino-based chaotic secure communication system using multi-directional multiscroll chaotic oscillators, Nonlinear Dyn. 87 (2017) 2203-2217.

29. B. Kumari and N. Gupta, Realization of chaotic circuits using lambda diode, J. Circuits, Syst. Comput. 26 (2017) 1750189.

30. J. C. Sprott and S. J. Linz, Algebraically simple chaotic flows, Int. J. Chaos Theory Appl. 5 (2000) 1-20.

31. P. Bartissol and L. Chua, The double hook, IEEE Trans. Circuits Syst. 35 (1988) 15121522.

32. J. C. Sprott, S. Jafari, V. T. Pham and Z. S. Hoseini, A chaotic system with a single unstable node, Phys. Lett. A 379 (2015) 2030-2036.

33. Z. Wei, J. C. Sprott and H. Chen, Elementary quadratic chaotic flows with a single nonhyperbolic equilibrium, Phys. Lett. A 377 (2015) 2184-2187.

34. S. Jafari, J. C. Sprott and S. M. R. H. Golpayegani, Elementary quadratic chaotic flows with no equilibria, Phys. Lett. A 377 (2013) 699-702.

35. Z. Wei, Dynamical behaviors of a chaotic system with no equilibria, Phys. Lett. A 376 (2011) 102-108.

36. F. R. Tahir, S. Jafari, V. T. Pham, C. Volos and X. Wang, A novel no-equilibrium chaotic system with multiwing butterfly attractors, Int. J. Bifurcation Chaos 25 (2015) 1550056.

37. V. T. Pham, C. Volos, S. Jafari, Z. Wei and X. Wang, Constructing a novel no-equilibrium chaotic system, Int. J. Bifurcation Chaos 24 (2014) 1450073.

38. Z. Wang, A. Akgul, V. T. Pham and S. Jafari, Chaos-based application of novel noequilibrium chaotic system with coexisting attractors, Nonlinear Dyn. 89 (2017) 18771887.

39. V. T. Pham, S. Vaidyanathan, C. Volos, S. Jafari and S. T. Kingni, A no-equilibrium hyperchaotic system with a cubic nonlinear term, Optik 127 (2016) 3259-3265.

40. Z. Wang, S. Cang, E. Oketch Ochola and Y. Sun, A hyperchaotic system without equilibrium, Nonlinear Dyn. 69 (2012) 531-537.

41. V. T. Pham, X. Wang, S. Jafari, C. Volos and T. Kapitaniak, From Wang-Chen system with only one stable equilibrium to a new chaotic system without equilibrium, Int. J. Bifurcation Chaos 27 (2017) 1750097.

42. M. Molaie, S. Jafari, J. C. Sprott, S. M. R. H. Golpayegani, Simple chaotic flows with one stable equilibrium, Int. J. Bifurcation Chaos 23 (2013) 1350188.

43. X. Wang and G. Chen, A chaotic system with only one stable equilibrium, Commun. Nonlinear Sci. Numer. Simul. 17 (2012) 1264-1272.

44. Z. Wei and Q. Yang, Dynamical analysis of the generalized Sprott C system with only two stable equilibria, Nonlinear Dyn. 68 (2012) 543-554.

45. S. Jafari and J. C. Sprott, Simple chaotic flows with a line equilibrium, Chaos, Solitons Fractals 57 (2013) 79-84.

46. C. Li, J. C. Sprott and W. Thio, Bistability in a hyperchaotic system with a line equilibrium, J. Exp. Theor. Phys. 118 (2014) 494-500.

47. S. Jafari, J. C. Sprott and M. Molaie, A simple chaotic flow with a plane of equilibria, Int. J. Bifurcation Chaos 26 (2016) 1650098.

48. J. Petrzela and T. Gotthans, New chaotic dynamical system with a conic-shaped equilibrium located on the plane structure, Appl. Sci. 7 (2017) 976-988.

49. T. Gotthans and J. Petrzela, New class of chaotic systems with circular equilibrium, Nonlinear Dyn. 81 (2015) 1143-1149. 
50. T. Gotthans, J. C. Sprott and J. Petrzela, Simple chaotic flow with circle and square equilibrium, Int. J. Bifurcation Chaos 26 (2016) 1650137.

51. S. T. Kingni, V. T. Pham, S. Jafari and P. Woafo, A chaotic system with an infinite number of equilibrium point located on a line and on a hyperbola and its fractional-order form, Chaos, Solitons Fractals 99 (2017) 209-218.

52. K. Barati, S. Jafari, J. C. Sprott and V. T. Pham, Simple chaotic flows with a curve of equilibria, Int. J. Bifurcation Chaos 26 (2016) 1650034.

53. V. T. Pham, C. Volos, T. Kapitaniak, S. Jafari and X. Wang, Dynamics and circuit of a chaotic system with curve of equilibrium points, Int. J. Electron. 6 (2017) 1-13.

54. S. Jafari, J. C. Sprott, V. T. Pham, C. Volos and C. Li, Simple chaotic 3D flows with surfaces of equilibria, Nonlinear Dyn. 86 (2016) 1349-1358.

55. V. T. Pham, C. Volos, S. Jafari and T. Kapitaniak, A novel cubic-equilibrium chaotic system with coexisting hidden attractors: Analysis and circuitry implementation, J. Circuits, Syst. Comput. 27 (2017) 1850066.

56. X. Wang and G. Chen, Constructing a chaotic system with any number of equilibria, Nonlinear Dyn. 71 (2013) 429-436.

57. V. T. Pham, S. Jafari, C. Volos and T. Kapitaniak, Different families of hidden attractors in a new chaotic system with variable equilibrium, Int. J. Bifurcation Chaos 27 (2017) 1750138.

58. V. T. Pham, C. Volos, S. Jafari, S. Vaidyanathan, T. Kapitaniak and X. Wang, A chaotic system with different families of hidden attractors, Int. J. Bifurcation Chaos 26 (2016) 1650139.

59. D. Dudkowski, S. Jafari, T. Kapitaniak, N. V. Kuznetsov, G. A. Leonov and A. Prasad, Hidden attractors in dynamical systems, Phys. Rep. 637 (2016) 1-50.

60. G. A. Leonov, N. V. Kuznetsov and V. I. Vagaitsev, Localization of hidden Chua's attractors, Phys. Lett. A 375 (2011) 2230-2233.

61. G. A. Leonov, N. V. Kuznetsov and V. I. Vagaitsev, Hidden attractors in smooth Chua system, Physica D 241 (2012) 1482-1486.

62. J. Petrzela and M. Guzan, Analog implementations of dynamical systems with equilibria degenerated into plane objects, Proc. Int. Conf. Telecommunications and Signal Processing (Wien, 2016), pp. 243-249.

63. J. C. Sprott, Chaos and Time Series Analysis (Oxford University Press, Oxford, 2003), p. 507.

64. S. Kodba, M. Perc and M. Marhl, Detecting chaos from a time series, Euro. J. Phys. 26 (2005) 205-215.

65. A. Wolf, J. B. Swift, H. L. Swinney and J. A. Vastano, Determining Lyapunov exponents from a time series, Physica D 16 (1985) 285-317.

66. J. Petrzela, Optimal piecewise-linear approximation of the quadratic chaotic dynamics, Radioengineering 21 (2012) 20-28.

67. T. Gotthans, J. Petrzela and Z. Hrubos, Advanced parallel processing of Lyapunov exponents verified by practical circuit, Proc. Int. Conf. Radioelektronika (Brno, 2011), pp. $405-408$.

68. L. G. de la Fraga and E. Tlelo-Cuautle, Optimizing the maximum Lyapunov exponent and phase space portraits in multi-scroll chaotic oscillators, Nonlinear Dyn. 76 (2014) 1503-1515.

69. J. Petrzela, T. Gotthans and Z. Hrubos, Behavior identification in the real electronic circuits, Proc. Int. Conf. Mixed Design of Integrated Circuits and Systems (Lodz, 2011), pp. $438-441$. 
70. Ch. Li, J. C. Sprott, Chaotic flows with a single nonquadratic term, Phys. Lett. A $\mathbf{3 7 8}$ (2014) 178-183.

71. J. C. Sprott, Symmetric time-reversible flows with a strange attractor, Int. J. Bifurcation Chaos 25 (2015) 1550078.

72. D. Biolek, R. Senani, V. Biolkova and Z. Kolka, Active elements for analog signal processing: Classification, review, and new proposals, Radioengineering 17 (2008) 15-32.

73. C. Sanchez-Lopez, R. Trejo-Guerra, J. M. Munoz-Pacheco and E. Tlelo-Cuautle, N-scroll chaotic attractors from saturated functions employing CCII+s, Nonlinear Dyn. 61 (2010) 331-341.

74. A. S. Elwakil and M. P. Kennedy, Improved implementation of Chua's chaotic oscillator using current feedback op amp, IEEE Trans. Circuits Syst. I, Fundam. Theory Appl. 47 (2000) 76-79.

75. J. M. Munoz-Pacheco, E. Tlelo-Cuautle, I. Toxqui-Toxqui, C. Sanchez-Lopez and R. Trejo-Guerra, Frequency limitations in generating multi-scroll chaotic attractors using CFOAs, Int. J. Electron. 101 (2014) 1559-1569.

76. T. M. Hassan and S. A. Mahmoud, New CMOS DVCC realization and applications to instrumentation amplifier and active-RC filters, $A E U$ - Int. J. Electron. Commun. 64 (2010) 47-55.

77. A. Imran, D. Arora and R. Kumar, Dual DVCC based voltage-mode digitally programmable biquadratic filter, Circuits Syst. 5 (2014) 1-6.

78. J. Petrzela, Utilization of DVCC element for modeling driven nonlinear dynamics, Proc. Int. Conf. Telecommunications and Signal Processing (Prague, 2015), pp. 1-5.

79. A. Lahiri and A. Chowdhury, A novel first-order current-mode all-pass filter using CDTA, Radioengineering 18 (2009) 300-305.

80. A. U. Keskin and D. Biolek, Current mode quadrature oscillator using current differencing transconductance amplifiers (CDTA), IEE Proc. - Circuits, Devices Syst. 153 (2006) 214-218.

81. D. Prasad, D. R. Bhaskar and A. K. Singh, New grounded and floating simulated inductance circuits using current differencing transconductance amplifiers, Radioengineering 19 (2010) 194-197.

82. J. C. Sprott, Simplest dissipative chaotic flow, Phys. Lett. A 228 (1997) 271-274.

83. R. Karthikeyan, A. Akgul, S. Jafari, A. Karthikeyan and I. Koyuncu, Chaotic chameleon: Dynamical analysis, circuit implementation, FPGA design and fractional-order form with basic analysis, Chaos, Solitons Fractals 103 (2017) 476-487. 